THE COMPUTER MODEL HYDROTHERM, A THREE-DIMENSIONAL FINITEDIFFERENCE MODEL TO SIMULATE GROUND-WATER FLOW AND HEAT TRANSPORT IN THE TEMPERATURE RANGE OF 0 TO $1,200^{\circ} \mathrm{C}$ By D.O. Hayba and S.E. Ingebritsen

U.S. GEOLOGICAL SURVEY

Water-Resources Investigations Report 94-4045

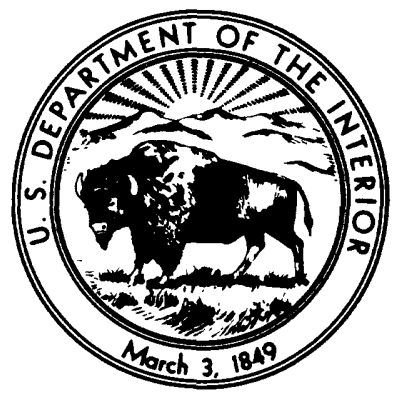




\section{U.S. DEPARTMENT OF THE INTERIOR \\ BRUCE BABBITT, Secretary}

\section{U.S . GEOLOGICAL SURVEY}

Gordon P. Eaton, Director

Any use of trade, product, or firm names is for descriptive purposes only and does not imply endorsement by the

U.S. Government

For additional information write to:

D.O. Hayba

U.S. Geological Survey

959 National Center

Reston, VA 22092
Copies of this report can be purchased from:

U.S. Geological Survey

Earth Science Information center Open-File Reports Section

Box 25286, MS 517

Denver Federal Center

Denver, CO 80225

- III- 


\section{CONTENTS}

Page

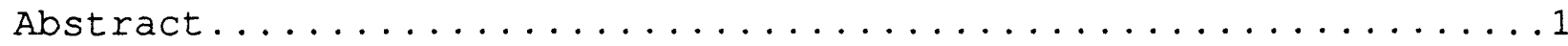

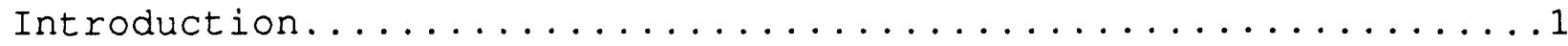

Status of geothermal modeling...................

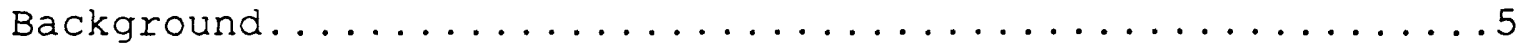

Purpose and scope........................

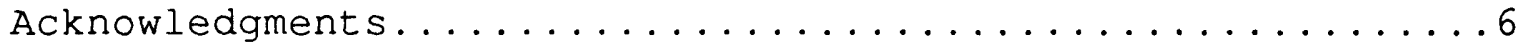

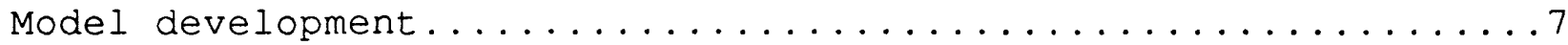

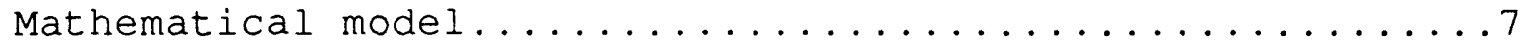

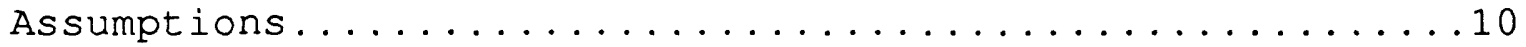

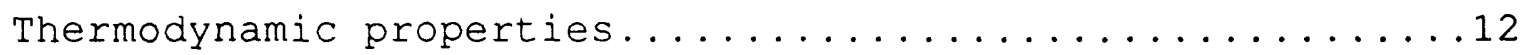

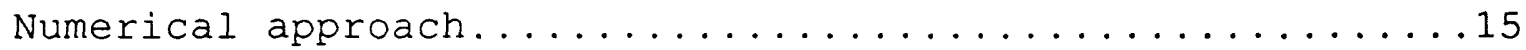

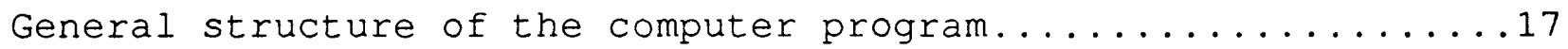

Practical considerations for model application...........23

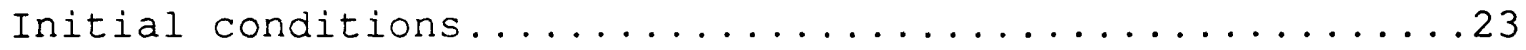

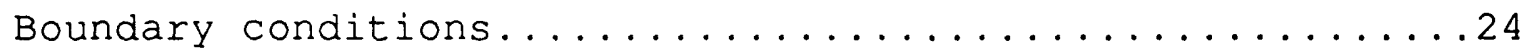

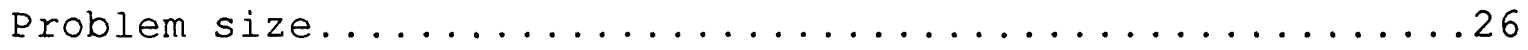

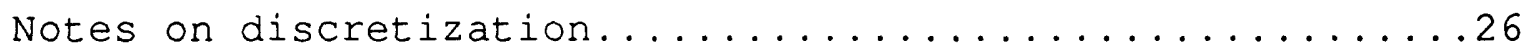

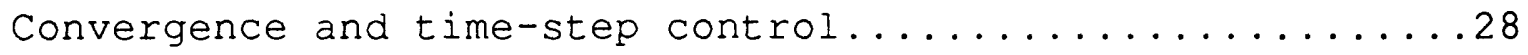

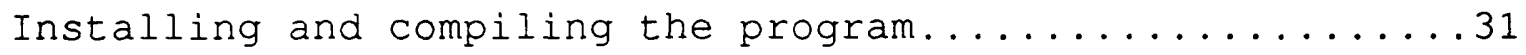

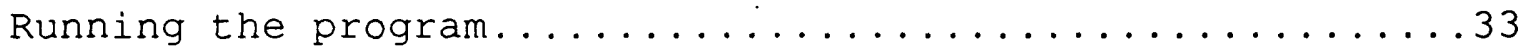

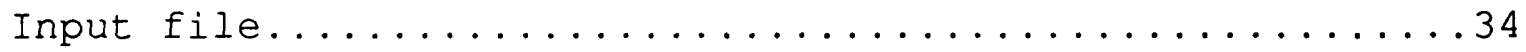

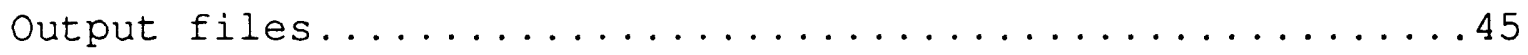

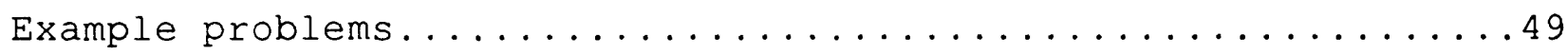

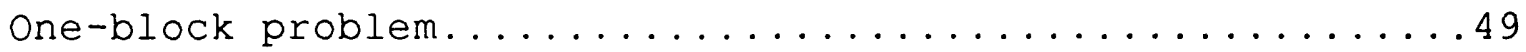

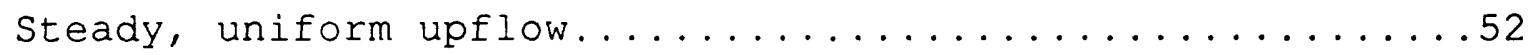

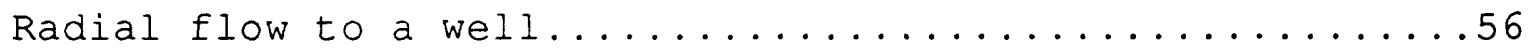

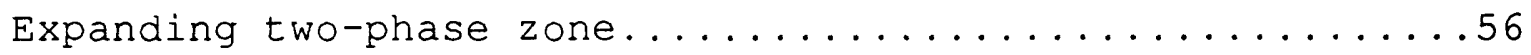

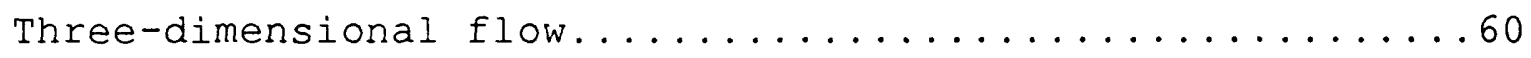

References................................64 
Attachment A: Description of selected program variables....68 Attachment B-H: HYDROTHERM input files for:

B: One-block problem: Constant enthalpy.......71

C: One-block problem: Constant pressure.......73

D: Steady, uniform upflow:

Constant-flux lower boundary...........75

E: Steady, uniform upflow:

Constant-temperature lower boundary.......77

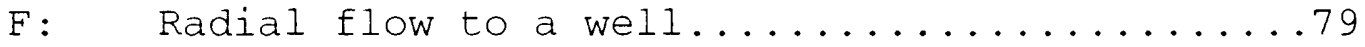

G: Expanding two-phase zone problem..........81

$\mathrm{H}: \quad$ Three-dimensional flow problem.............83

\section{ILLUSTRATIONS}

Figure 1. Pressure-enthalpy diagram for pure water........4

Figure 2. Example finite-difference grids for simulating

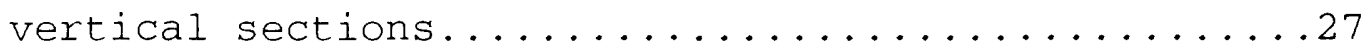

Figure 3. Temperature solutions for steady upflow at various

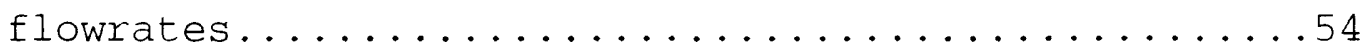

Figure 4. Pressure and saturation solutions for radial flow

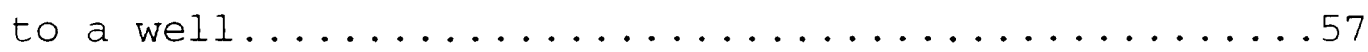

Figure 5. Pressure and saturation solutions for the expanding

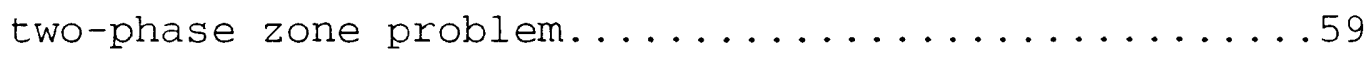

Figure 6. Pressure and saturation solutions for the three-

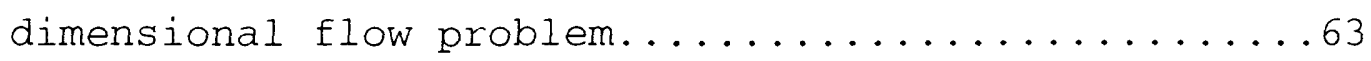




\section{TABLES}

Page

Table 1. Outline of HYDROTHERM program structure.........18

Table 2. List of HYDROTHERM subroutines and functions for calculating fluid properties and relative

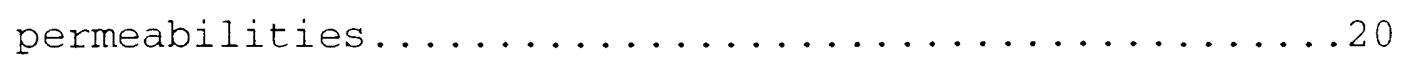

Table 3. Auxiliary files used in HYDROTHERM..............21

Table 4. Factors that control the time step in HYDROTHERM....30

Table 5. Complete list of HYDROTHERM program files........32

Table 6. List of HYDROTHERM output files.............46

Table 7. Results from application of HYDROTHERM to

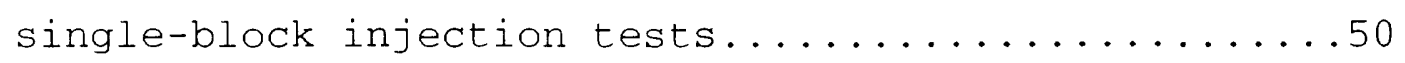

Table 8. Parameter values for the steady upflow problem.....55

Table 9. Specifications for the three-dimensional flow

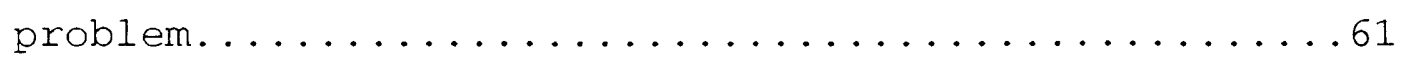




\section{CONVERSION FACTORS}

Conversion factors for units used in this report are listed below (HYDROTHERM uses cgs units):

multiply

\section{Length}

centimeter $(\mathrm{cm})$

meter $(\mathrm{m})$

kilometer $(\mathrm{km})$

Mass

gram (g)

Pressure

dyne

dyne

dyne by

to obtain

2.540

3.281

0.6214

0.03527 ounces (oz)

$1.0 \times 10^{-6}-6$ bars (b)

1. $013 \times 10^{-6}$ atmospheres (atm)

1. $451 \times 10^{-5}$

inches (in)

feet ( $f t$ )

miles (mi) $\left(1 \mathrm{~b} / \mathrm{in}^{2}\right)$

\section{Energy}

erg

erg

milliwatt per square meter

$1.0 \times 10^{-7}$

2. $389 \times 10^{-8}$

0.02389 $\left(\mathrm{mW} / \mathrm{m}^{2}\right)$

Thermal-conductivity units erg per second centimeter kelvin (erg/ $\left.\mathrm{s}^{\circ} \mathrm{cm}^{\circ} \mathrm{K}\right)$ erg per second centimeter $\mathrm{kelvin}\left(\mathrm{erg} / \mathrm{s}^{\circ} \mathrm{cm}^{\circ} \mathrm{K}\right)$

$1.0 \times 10^{-5}$

$2.389 \times 10^{-5}$ joule (J) calories (cal) heat-flow units (hfub

( $\left.1 \mathrm{hfu}=1 \mathrm{ucal} / \mathrm{cm}^{2} \cdot \mathrm{s}\right)$

\section{Permeability}

centimeter squared $\left(\mathrm{cm}_{2}^{2}\right)$ centimeter squared $\left(\mathrm{cm}^{2}\right)$
$1.0 \times 10_{7}^{-4}$

1. $0 \times 10^{7}$ watt per meter degree Kelvin $\left(\mathrm{W} / \mathrm{m}^{\circ} \mathrm{K}\right)$

thermal-conductivity $\operatorname{units}_{1 \times 10^{-}}(\mathrm{tcu})\left(1 \mathrm{mcal} / \mathrm{cm} \cdot \mathrm{s}^{\cdot 0} \mathrm{C}\right)$

meters squared $\left(\mathrm{m}^{2}\right)$ darcies (D)

For conversion of degrees Celsius $\left({ }^{\circ} \mathrm{C}\right)$ to degrees Fahrenheit $\left({ }^{\circ} \mathrm{F}\right)$, use the formula ${ }^{\circ} \mathrm{F}=9 / 5^{\circ} \mathrm{C}+32$.

For conversion of degrees Celsius $\left({ }^{\circ} \mathrm{C}\right)$ to kelvins $(\mathrm{K})$, use the formula $\mathrm{K}={ }^{\circ} \mathrm{C}+273.15$ 


\section{LIST OF SYMBOLS}

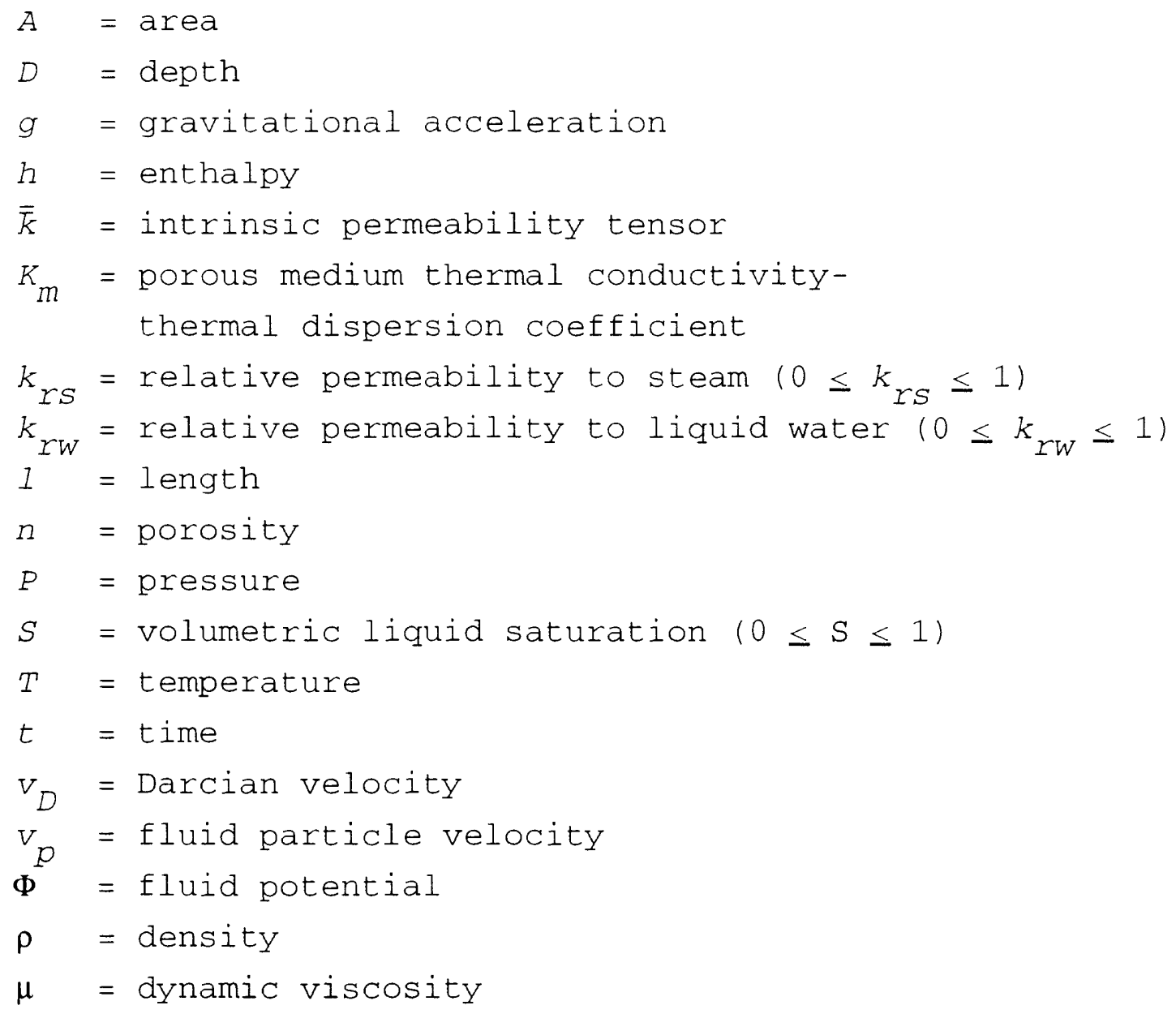

\section{Subscripts}

$f$ designates the fluid in place (single phase or two-phase mixture)

$i, j, k x_{-}, y^{-}$, and $z$-direction discretization indices (vertical section is $x, z$ )

$r$ refers to the porous medium (rock)

sat refers to "saturated" conditions (steam and liquid water both present)

$s$ refers to steam

$w \quad$ refers to liquid water

Other notation is defined where it is used in the text. 


\title{
THE COMPUTER MODEL HYDROTHERM, A THREE-DIMENSIONAL FINITE- DIFFERENCE MODEL TO SIMULATE GROUND-WATER FLOW AND HEAT TRANSPORT IN THE TEMPERATURE RANGE OF 0 TO $1,200^{\circ} \mathrm{C}$
}

By D.O. Hayba and S.E. Ingebritsen

\begin{abstract}
Quantitative modeling of the deep parts of magmatichydrothermal systems has been limited by the lack of publicly available, documented computer models for multiphase, hightemperature flow. This report documents HYDROTHERM, a finitedifference model for three-dimensional, multiphase flow of pure water and heat over a temperature range of 0 to 1,200 degrees Celsius and a pressure range of 0.5 to 10,000 bars. HYDROTHERM is an extension of multiphase geothermal models developed by the U.S. Geological Survey in the 1970's. It solves numerical approximations to mass- and energy-balance equations that are posed in terms of pressure and enthalpy. Major assumptions are that the rock matrix can be treated as a porous medium; that water and rock are in thermal equilibrium; and that capillary pressure is negligible.
\end{abstract}

\section{INTRODUCTION}

Transport processes in magmatic-hydrothermal systems involve single- or two-phase flow of fluids at temperatures ranging from 0 to greater than $1,000^{\circ} \mathrm{C}$ and pressures ranging up to several kilobars. Simulating fluid flow in these systems is computationally difficult because the properties of water change substantially over the relevant pressure-temperature range, especially in the vicinity of the critical point. Quantitative flow modeling of magmatic-hydrothermal systems has been limited by existing computer models that can simulate either 1) only 
single-phase flow, or 2) two-phase flow at subcritical conditions.

\section{Status of geothermal modeling}

The HYDROTHERM model described in this report is the first publicly documented, multiphase geothermal model that handles near-critical and supercritical temperatures. Previously, most users of geothermal models have had to choose between models for multiphase, pure-water (or water and gas) systems with a temperature range of about $0-350^{\circ} \mathrm{C}$ and models for single-phase pure-water systems with a temperature range of about $0-1,000^{\circ} \mathrm{C}$. Most of the multiphase, subcritical models have been developed by reservoir-engineering groups and are designed to handle the range of conditions encountered in geothermal-reservoir development (for example, Pruess, 1991a). Most of the single-phase hightemperature models were developed by geoscience groups, and a major application has been the quantitative description of heat transfer associated with cooling plutons (for example, Norton and Knight, 1977; Cathles, 1977). Both the lower-temperature multiphase and higher-temperature single-phase models have generally assumed that the circulating fluid is pure water or water and noncondensible gas.

Higher-temperature multiphase models, such as HYDROTHERM, are needed for problems involving cooling plutons, crustal-scale heat transfer, or the deep zones of major hydrothermal systems such as The Geysers, California. In volcanic systems with shallow intrusions (less than 3-to 4-km depth), two-phase steamliquid water flow is probably common. In such systems, the subcritical two-phase models cannot simulate the necessary range in temperature, and the single-phase models cannot mimic important multiphase processes. The temperature range that HYDROTHERM can simulate exceeds that of a silicic magmatichydrothermal system (perhaps $0-850^{\circ} \mathrm{C}$ ) and is approximately equal 
to that of a basaltic magmatic-hydrothermal system (perhaps 0$1,200^{\circ} \mathrm{C}$ ). HYDROTHERM allows simulation of multiphase processes, including (for example) phase separation, heat transfer by the heat-pipe mechanism (White and others, 1971), and boiling/condensation that buffers pressure transmission (Grant and Sorey, 1979) and limits near-surface temperatures to the boiling-point curve.

Unlike most other high-temperature models (Furlong and others, 1991, p. 472f), HYDROTHERM allows for fluid flow due to changes in fluid and pore volumes within model elements. Neglecting these driving forces for flow is a reasonable assumption in a high-permeability environment. However, given heating rates typical of metamorphic environments, it is a poor assumption for permeabilities less than about $10^{-16} \mathrm{~m}^{2}$ (Hanson, 1992 ).

One of the major obstacles to development of multiphase, high-temperature models is the radical variation in fluid properties near the critical point. For simulators that use pressure and temperature as dependent variables, the singularity in fluid heat capacity at the critical point is a particularly difficult problem. A simple pressure-temperature formulation cannot be used in multiphase models, because the pressuretemperature pair does not specify saturation. Some multiphase codes, such as TOUGH2 (Pruess, 1991a), switch variables, solving for pressure and temperature in the single-phase region and pressure and saturation in the two-phase region. In developing GEOTHER, the predecessor of HYDROTHERM, Faust and Mercer (1977, 1979a) chose pressure and enthalpy as dependent variables, because they uniquely specify the thermodynamic state of the fluid under both single- and two-phase conditions (fig. 1 ). Their choice of variables also greatly facilitated the extension of HYDROTHERM to near- and super-critical conditions. 


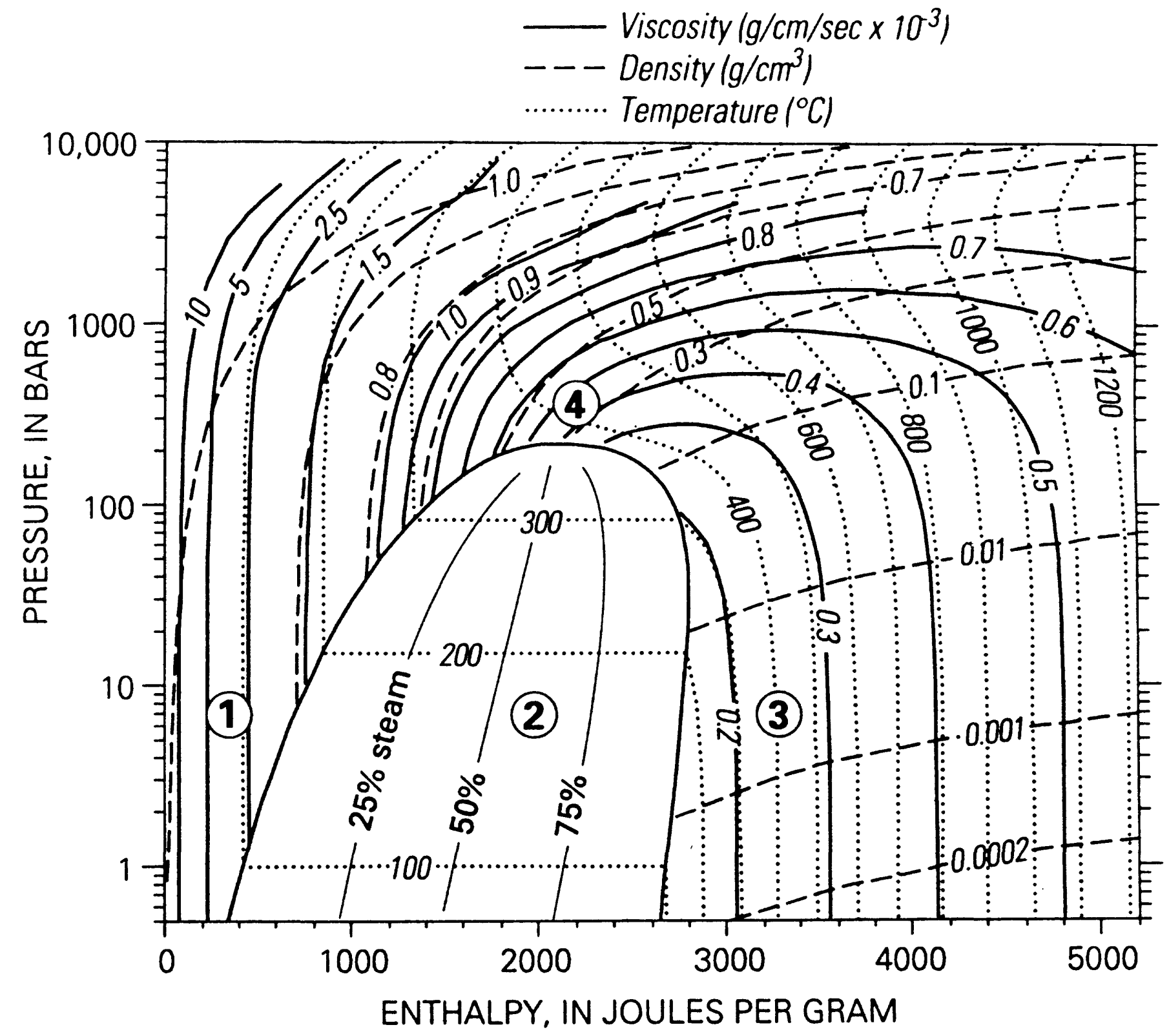

Figure 1. Pressure-enthalpy diagram for pure water, showing contours of equal temperature, density, viscosity, and mass proportion of steam. The thermodynamic regions are (1) compressed liquid water (2) two-phase (3) superheated steam and (4) supercritical fluid. The saturated enthalpies of steam and liquid water define the boundaries of the two-phase region (2), and meet at the critical point (220.55 bars and $2086.0 \mathrm{~J} / \mathrm{g}$ ). 
Roberts and others (1987) described another extended version (to 1,000 $\mathrm{C}$ and 1,000 bars) of the Faust and Mercer model, but their documentation and source code have not entered the public domain. The approach of Roberts and others (1987) was to divide the thermodynamic range into a number of subregions and develop iterative equations of state for each subregion.

Although HYDROTHERM is capable of simulating hightemperature, multiphase processes, it assumes that the circulating fluid is pure water. The next step towards a more global simulator will be to develop a high-temperature, multiphase, multicomponent model.

\section{Background}

HYDROTHERM is a descendent of the multiphase geothermal models developed at the U.S. Geological Survey in the 1970's (Faust and Mercer, 1977, 1979a, 1979b, 1982). After C.R. Faust and J.W. Mercer left the Geological Survey, Faust restructured an earlier version of the GEOTHER documentation (Faust and Mercer, 1982) so that it would be consistent with Nuclear Regulatory Commission guidelines (see Intera, 1983). Most of the important modifications to the Faust and Mercer (1982) model are summarized in this report; others were described by Hayba (1993).

We have extensively modified and expanded the threedimensional GEOTHER model described by Faust and Mercer (1982). Important revisions include extending the temperature range from $10-300^{\circ} \mathrm{C}$ to $0-1,200^{\circ} \mathrm{C}$, extending the pressure range from $1-175$ bars to 0.5-10,000 bars, modularizing the program architecture, adding provisions for spatial and temporal variation of porousmedium properties, automating the time-step control, and making the convergence criteria more rigorous. We also replaced the regression equations for density, viscosity, and temperature with a more accurate routine that uses bicubic interpolation of a 
lookup table. Finally, we have extensively revised the input and output formats to make the program easier to use.

\section{Purpose and scope}

This report documents the computer program HYDROTHERM, a three-dimensional, multiphase finite-difference model that simulates the flow of pure water and heat in a porous medium. The model allows for spatial and temporal variations of medium properties such as permeability, porosity, and thermal conductivity, and incorporates pure-water equations of state that cover the temperature range of $0-1,200^{\circ} \mathrm{C}$ and pressures from 0.5 bars to 10 kbars. HYDROTHERM cannot treat salinity, but its modular architecture facilitates continued revision, and allows for the future addition of solute transport.

This report describes the mathematical and numerical models upon which the computer program is based, presents an overview of the general structure of the program, discusses practical considerations for model application, and includes a set of example problems that demonstrate both the thermodynamic range and the multi-dimensional performance of the model.

\section{Acknowledgments}

We thank Wen-yue Xu of the Georgia Institute of Technology for providing routines that helped us to generate some of the tabulated thermodynamic data used in HYDROTHERM, and Hedeff Essaid and Elizabeth Rowan for numerous discussions and comments that have resulted in a improved report. 
With the exception of the extended equations of state, relatively minor changes have been made to the mathematical model and numerical approach described by Faust and Mercer (1979a, 1979b, 1982). Thus this section is relatively brief, and the reader is encouraged to refer to the earlier papers for further details.

\section{Mathematical model}

The governing equations are expressions of mass and energy conservation that are posed in terms of pressure and enthalpy. Unlike pressure and temperature, using pressure and enthalpy as the dependent variables uniquely specifies the thermodynamic state of the fluid under both single- and two-phase conditions (fig. 1). The pressure-enthalpy formulation also avoids some difficult problems associated with the critical point, including the singularity in heat capacity (discussed above) and divergence of partial derivatives of $\rho(P, T)$ to $\pm \infty$ (Johnson and Norton, 1991, p.543).

The mass- and energy-balance equations are

$$
\begin{aligned}
\partial\left(n \rho_{f}\right) / \partial t & -\nabla \cdot\left[\bar{k} k_{r S} \rho_{S} / \mu_{S} \bullet\left(\nabla P-\rho_{S} g \nabla D\right)\right] \\
& -\nabla \cdot\left[\bar{k} k_{r W} \rho_{W} / \mu_{W} \bullet\left(\nabla P-\rho_{W} g \nabla D\right)\right]-q_{m}=0
\end{aligned}
$$

and

$$
\begin{aligned}
\partial / \partial t\left[n \rho_{f} h_{f}+(1-n) \rho_{r} h_{r}\right] & -\nabla \cdot\left[\bar{k} k_{r S} \rho_{S} h_{S} / \mu_{S} \cdot\left(\nabla P-\rho_{S} g \nabla D\right)\right] \\
& -\nabla \cdot\left[\bar{k} k_{r W} \rho_{W} h_{W} / \mu_{W} \cdot\left(\nabla P-\rho_{W} g \nabla D\right)\right] \\
& -\nabla \cdot K_{m} \nabla T-q_{h}=0
\end{aligned}
$$


respectively. The density $\left(\rho_{f}\right)$ and enthalpy $\left(h_{f}\right)$ of the fluid in place are defined by

and

$$
\rho_{f}=S_{W} \rho_{W}+S_{S} \rho_{S}
$$

$$
h_{f}=\left[h_{W} S_{W} \rho_{W}+h_{S} S_{S} \rho_{S}\right] /\left[S_{W} \rho_{W}+S_{S} \rho_{S}\right]
$$

where $S_{W}$ and $S_{S}$ are volumetric liquid and steam saturations, respectively, and

$$
S_{W}+S_{S}=1
$$

with $S_{W}=1$ in the compressed-water region and $S_{S}=1$ in the superheated-steam region.

Extending HYDROTHERM to supercritical conditions created some problems with the definition of volumetric saturation and the liquid water/steam nomenclature. Because the distinction between liquid and steam disappears above the critical point, the values assigned to the saturation variables become somewhat arbitrary. However, when there is flow from a supercritical finite-difference block to a subcritical block (liquid, twophase, or superheated-steam), the averaged density and viscosity values for interblock flow depend on the saturation value assigned to the supercritical block. We enforced consistent averaging by treating supercritical blocks as though they contain two phases with identical properties (density, viscosity, relative permeability, and saturation).

The source/sink terms $q_{m}$ and $q_{h}$ are defined as

and

$$
q_{m}=q_{S}+q_{W}
$$




$$
q_{h}=q_{S} h_{S}+q_{W} h_{W}
$$

with $q$ denoting a mass flowrate (g/s) (Faust and Mercer, 1979a). Sources and sinks include conductively heated blocks along the base of the finite-difference grid and those blocks affected by pumping or injection from a well. For wells, fluxes of mass and energy are allocated along vertical columns, on the basis of fluid mobility and the permeability and thickness of userspecified open intervals.

The first term of (1) is a mass storage term and the second and third terms represent the divergence of mass flux of steam and liquid water, respectively. The first term of (2) is an energy storage term, and the second and third terms represent advective transport of heat by steam and liquid water, respectively. They are equivalent to the second and third terms of (1) multiplied by the enthalpy of the appropriate phase. The fourth term of (2) represents conductive heat transport. Faust and Mercer (1979a) expanded the temperature gradient in this term to

$$
\left[(\partial T / \partial P){ }_{h} \nabla P+(\partial T / \partial h){ }_{P} \nabla h\right] .
$$

We determined that this expansion was unnecessary, and that it caused convergence failures in some cases (usually at a phase transition). With the new representation of the equations of state, HYDROTHERM can calculate the temperature as a function of pressure and enthalpy very accurately.

Equations (1)-(7) alone are not sufficient to describe a geothermal system. Further constitutive relations are needed, and appropriate boundary conditions must be specified. The assumptions involved in derivation of the governing equations and constitutive relations are outlined below, and boundary conditions are discussed in a later section. 


\section{Assumptions}

The important assumptions used in the derivations of equations (1) and (2) are as follows (see also Faust and Mercer, 1979a):

1) The fluid is pure water.

2) A two-phase form of Darcy's Law is valid.

3) Capillary-pressure effects are negligible.

4) Relative permeabilities are non-hysteretic functions of liquid volume saturation.

5) Rock and water are in local thermal equilibrium.

6) Heat transfer by dispersion and radiation are negligible.

7) Porosity is a linear function of pressure, given by $n=n_{i n}+\alpha\left(P-P_{i n}\right)$, where $n_{i n}$ and $P_{i n}$ are initial porosity and pressure, respectively, and $\alpha$ is a vertical compressibility coefficient.

8) Rock enthalpy is a linear function of temperature.

9) Porosity, intrinsic permeability, and thermal conductivity can vary in space and time, whereas rock density and specific heat of rock are constant.

Pure water - As Faust and Mercer (1979a) pointed out, dissolved solid and gas concentrations in many geothermal areas are low enough that fluid properties do not differ significantly from those of pure water. However, the pure-water assumption is clearly inappropriate for systems involving seawater or magmaticbrine components. We anticipate that future versions of HYDROTHERM will be extended to treat two-component multiphase systems .

Capillary pressure - It is difficult to estimate appropriate capillary-pressure functions for hydrothermal systems, as relevant data are limited. The surface tension of water decreases with temperature, so capillary-pressure effects should become relatively less important at high temperatures. The 
HYDROTHERM formulation assumes that the fluid pressures in liquid and steam phases are equal, but the relative-permeability functions implicitly recognize capillary effects by allowing for residual (immobile) water and steam saturations. Like the purewater assumption, the assumption that capillary pressure is negligible greatly simplifies solution of the governing equations.

\section{Rock-water thermal equilibrium; thermal dispersion - The} assumption that rock and water are in local thermal equilibrium is commonly made in geothermal reservoir engineering and is reasonable if fluid flow is relatively slow or steady; it breaks down during rapid transients. The assumption that thermal dispersion is negligible is also common; because heat conduction is a relatively efficient process, the influence of thermal dispersion on heat-transfer problems tends to be much less important than that of hydrodynamic dispersion on solutetransport problems. Faust and Mercer (1979a) suggested that the coefficient $K_{m}$ could be regarded as a lumped thermal conductivity-thermal dispersion coefficient. That approach is incomplete at best; it does not allow dispersion to vary with fluid velocity and other relevant parameters.

\section{Heat radiation and temperature-dependent rock properties -} The assumption of negligible heat radiation is potentially problematic for high-temperature applications, as are several other aspects of the formulation. Radiative heat transfer may become significant at temperatures $>600^{\circ} \mathrm{C}$. Also, over the extended $0-1,200^{\circ} \mathrm{C}$ temperature range the thermal conductivity and heat capacity of many rocks vary significantly with temperature. As noted above, porous-medium thermal conductivity is currently allowed to vary in space and with time (but not directly with temperature), and rock heat capacity is held constant. One approach to radiative transfer would be to treat $K_{m}$ as a lumped conduction-radiation coefficient. Explicitly incorporating 
temperature-dependent thermal conductivity and specific heat would be straightforward, and Sass and others (1992, p. 5,025) recently presented a convenient, general model for the variation of rock thermal conductivity with temperature.

\section{Thermodynamic properties}

In addition to the dependent variables, fluid pressure and enthalpy, the governing equations for HYDROTHERM require values for fluid densities, viscosities, and temperature. In the earlier version, GEOTHER, Faust and Mercer (1977, 1979a, 1982) used regression equations to calculate densities and temperature as functions of pressure and enthalpy, and viscosities as functions of temperature. Their regression equations have a maximum error of 0.5 percent over a temperature range of about $10-300^{\circ} \mathrm{C}$ and a pressure range of about 1-175 bars. We replaced these expressions, most of which break down in the range $300-$ $350^{\circ} \mathrm{C}$, with a lookup table that is interrogated by a bicubic interpolation routine. This method is very accurate and relatively fast, but the lookup table is large (about 2 megabytes). The coordinates of the table are pressure, with a range of 0.5-10,000 bars, and enthalpy, with a range of 1-5,200 $\mathrm{kJ} / \mathrm{kg}$. The temperature range corresponding to these pressure and enthalpy ranges is approximately $0-1,200^{\circ} \mathrm{C}$ (see fig. 1 ).

The lookup table contains approximately 4,500 grid points (pressure-enthalpy pairs), and actually consists of four subtables that mend together seamlessly. At each grid point, there are values for the density, temperature, and viscosity of water, as well as values for the gradients and cross derivatives of each of these properties with respect to pressure and enthalpy. There are no grid points in the two-phase region; properties of the coexisting phases are derived from the values for saturated liquid water and steam. The lookup table also excludes the region where temperatures are less than $0^{\circ} \mathrm{C}$ (low enthalpy/high pressure). 
The fluid density and temperature values in the table were calculated from the steam-table routines of Haar and others (1984). In 1982 the International Association for Properties of Steam (IAPS) provisionally accepted this formulation for the temperature range of $0-1,000^{\circ} \mathrm{C}$ and pressures up to $15 \mathrm{kbar}$, and Haar and others (1984) state that it adequately defines the properties of water to temperatures in excess of $2,000^{\circ} \mathrm{C}$.

Most of the tabulated viscosity values were obtained using the formulation by Watson and others (1980), which Sengers and Kamgar-Parsi (1984) re-evaluated using density values accepted by the 1982 IAPS agreement (Haar and others, 1984). Watson and others (1980) and Sengers and Kamgar-Parsi (1984) stated that their viscosity formulation is valid for the following range of temperatures and pressures:

$$
\begin{aligned}
& 0^{\circ} \mathrm{C} \leq T \leq 150{ }^{\circ} \mathrm{C}, \quad 0 \text { bar } \leq P \leq 5,000 \text { bar } \\
& 150^{\circ} \mathrm{C} \leq T \leq 600^{\circ} \mathrm{C}, \quad 0 \text { bar } \leq P \leq 3,500 \text { bar } \\
& 600^{\circ} \mathrm{C} \leq T \leq 900^{\circ} \mathrm{C}, \quad 0 \text { bar } \leq P \leq 3,000 \text { bar. }
\end{aligned}
$$

Watson and others (1980) indicated that calculated values deviate systematically from experimental values at higher pressures, but did not report the magnitude of the deviations. Because there are no available data beyond the limits of the sengers and Kamgar-Parsi (1984) formulation (hereafter SK-P), we needed to extrapolate viscosity values for pressures up to $10 \mathrm{kilobars}$ and temperatures up to $1,200^{\circ} \mathrm{C}$. Plots of temperature (or enthalpy) versus viscosity for various pressures suggest that one should be able to make moderately accurate extrapolations beyond the SK-P limits. The SK-P formulation itself appears to predict reasonable values for viscosity at high pressures for low temperatures $\left(<200^{\circ} \mathrm{C}\right)$ and at low pressures ( $<3$ kilobars) for high temperatures. However, at high pressures ( $>4$ kilobars) and temperatures $\left(>300^{\circ} \mathrm{C}\right)$ extrapolation of the SK-P relations leads to viscosities that appear unrealistically low. To construct our lookup table in this region, we estimated the viscosity of water at 10 kilobars from plots of pressure versus viscosity at 
selected enthalpies, and then interpolated values for viscosity between 10 kilobars and the SK-P limits. The estimated viscosities at 10 kilobars are probably accurate within a factor of two, and the errors should decrease at lower pressures. We expect that the overall uncertainty introduced by this extrapolation is relatively modest, especially when compared with uncertainties in intrinsic permeability at high pressures and temperatures.

HYDROTHERM uses a bicubic interpolation routine to derive thermodynamic properties from the lookup table (Press and others, 1986, p. 98-100). For any given pressure-enthalpy pair, the interpolation routine returns density, viscosity, and temperature values, as well as the gradients of those values with respect to pressure and enthalpy. These gradients are required by the Newton-Raphson iteration techniques employed in the numerical solution. The bicubic interpolation method exactly reproduces the thermodynamic values and specified derivatives at the grid points of the lookup table, and ensures that the values and their derivatives change continuously across grid-square boundaries within the table.

For the two-phase region, we use cubic splines (see Press and others, 1986, p. 86-89) to accurately describe the positions of the saturated-water and saturated-steam curves, calculate fluid properties, and obtain the necessary derivatives. The splines provide values for the enthalpy, density, temperature, and viscosity of saturated liquid water and steam as functions of pressure. There are 134 points along each spline, extending from 0.5 bars to the critical pressure (220.55 bars), with the greatest concentration of data near the critical point. The data for pressure, enthalpy, density, and temperature of saturated liquid water and steam are from Haar and others (1984), and the viscosity data are from Sengers and Kamgar-Parsi (1984). 


\section{Numerical approach}

The mass- and energy-balance equations are strongly coupled and highly nonlinear, because a number of the independent variables are functions of the dependent variables. The relative permeabilities, densities, and viscosities, in particular, vary widely with pressure and enthalpy. Faust and Mercer (1979b, 1982) described the numerical techniques used to solve finitedifference approximations to equations (1) and (2). Briefly, the nonlinear coefficients are treated using Newton-Raphson iteration. This leads to a system of linear equations that must be solved for each iteration. Each vertical cross section of the finite-difference grid is solved implicitly, and for a single vertical section the matrix equation is solved directly. Twodimensional areal models can be set up as vertical sections with gravitational acceleration set to zero, in order to take advantage of the direct solution. For a fully three-dimensional model, the solution technique is slice-successive overrelaxation embedded in the Newton-Raphson iteration.

Convergence is checked by calculating mass and energy balances. The 1982 version of the code, GEOTHER, used global mass- and energy-balance criteria. HYDROTHERM requires residual mass and energy for each finite-difference block to meet userspecified limits (defined in terms of $\mathrm{g} / \mathrm{s} / \mathrm{cm}^{3}$ and $\mathrm{ergs} / \mathrm{s} / \mathrm{cm}^{3}$ ).

The finite-difference formulation used by HYDROTHERM requires that values for some of the variables be estimated between, rather than at, block centers. Depending upon the variable, these interblock values may be assigned as an average of the values in adjacent blocks or as the value at the 'upstream' node. HYDROTHERM uses harmonic averages to calculate interblock transmissivities ( $k A / l)$ and conductivities $\left(K_{m} A / I\right)$. Fluid density and viscosity values are calculated as arithmetic averages. Like most numerical models describing fluid flow and heat transport, HYDROTHERM applies upstream weighting to fluid 
enthalpies and saturation-dependent functions such as relative permeabilities. Upstream weighting is defined by, for example,

$$
k_{r W, i+1 / 2}=\begin{aligned}
& k_{r W}\left(S_{W, i}\right) \text { if flow is from } i \text { to } i+1 \\
& k_{r W}\left(S_{W, i+1}\right) \text { if flow is from } i+1 \text { to } i .
\end{aligned}
$$

Without upstream weighting, numerical solutions of convectiondiffusion-type transport equations such as (2) are subject to oscillation and numerical diffusion when the first-order spatial derivative terms (advection) become large relative to the secondorder spatial derivative term (conduction) (Gladwell and Wait, 1979, p. 205f).

Previous (GEOTHER) versions of the code applied upstream weighting to fluid densities and viscosities, as well as fluid enthalpies and relative permeabilities, though the code description stated otherwise (Faust and Mercer, 1979b, p. 34). Use of "upstream" densities in the governing equations led to serious computational difficulties in cases where the "upstream" finite-difference block estimated on the basis of $\rho_{i}$ and $\rho_{i+1}$ was different than the "upstream" block estimated after equating $\rho_{i+1 / 2}$ with $\rho_{i}$ or $\rho_{i+1}$. Using upstream weighting solely for enthalpies and relative permeabilities greatly improves the stability of the algorithm.

HYDROTHERM also allows the use of weighted-average enthalpies and relative permeabilities when the potential for flow between blocks reaches a specified (low) value. Below this value, the upstream-weighting factor is scaled from 1.0 to 0.5 using a logarithmic function. The averaging option facilitates smooth, non-oscillatory changes in flow direction. 


\section{GENERAL STRUCTURE OF THE COMPUTER PROGRAM}

HYDROTHERM consists of an initialization routine followed by a time-stepping loop that contains nested loops for NewtonRaphson iteration and slice-successive overrelaxation. Table 1 illustrates the general structure of the computer program and the order in which most of the subroutines are called. Other subroutines, listed in table 2, calculate the fluid properties and relative permeabilities.

The initialization routine reads the input file for the problem and sets up the program for the first time step. Within each time step, the program uses Newton-Raphson iteration to solve the governing equations for each $x-z$ slice in the problem domain. The embedded slice-successive-overrelaxation loop is only required for three-dimensional problems. Newton-Raphson iterations continue until the mass and energy balances calculated for each block meet user-specified convergence criteria. The solution must also satisfy user-specified limits on changes in pressure, enthalpy, and saturation relative to the previous time step. If any changes exceed the specified limits, the program discards the solution and re-enters the Newton-Raphson loop with a smaller time step. If the solution is acceptable, HYDROTHERM compares the changes in saturation, pressure, and enthalpy with user-specified limits, estimates the feasible size for the next time step, and then begins the procedure again.

There are substantial differences between the stucture of HYDROTHERM and that of the 1982 version, GEOTHER. The GEOTHER code consisted of only 16 subroutines, and the program was difficult to modify because it lacked a modular architecture. The present version consists of 47 subroutines along with a number of other files (see table 3 ) that contain common-block and array-dimensioning statements. Some structural changes were necessary to accommodate the new automatic time-stepping 
Table 1. Outline of HYDROTHERM program structure

GDATA - read input data

GFILES - open input and output files

GTABLES - load lookup table into memory

PRINTOPT - read user-defined print options

READ - read input arrays

$\mathrm{RD} \quad$ - help read input arrays

WR - print input arrays

ENTHTEMP - compute initial enthalpy ( $H)$ from temperature

PRESS - calculate hydrostatic pressure ( $P$ )

SINK - read source/sink input data

TCALC - calculate permeability and conductivity terms

PHREG - determine thermodynamic region for a block

PRPTY - calculate fluid properties and derivatives

AVGPRPTY - calculate interblock fluid properties

WELLALLO - calculate source/sink distribution and derivatives

UPSTRE - determine upstream block for each interblock region

STORATIV - calculate initial mass and energy storativities

Print initial conditions

VELOCTY - calculate fluid-velocity vectors

OUTPLOT - write file for input to plotting program

PDATA - print results

WR - help print arrays

Initialize and update variables for time loop

TIMECNTL - write which factor controlled time-step size

RESET - reset arrays if previous Newton-Raphson iteration did not converge

PRPTY - calculate fluid properties and derivatives

AVGPRPTY - calculate interblock fluid properties

WELLALLO - calculate source/sink distribution and derivatives

UPSTRE - determine upstream block for interblocks

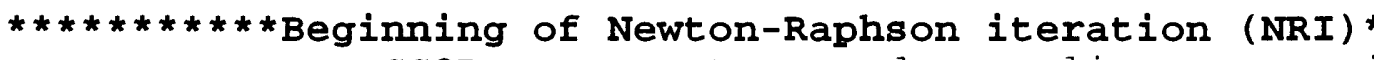

SSOR - set up and run slice-successive overrelaxation

FORMEQ - formulate matrix equations

SOLVE (1) - triangularize matrix

$* * * * * * *$ Beginning of Slice Successive Overrelaxation 1oop

SOLVE (2) - back substitution for each slice

$* * * * * * * * *$ End of Slice Successive Overrelaxation 1oop $* * * * * * * * * * *$ 
Table 1. Outline of HYDROTHERM program structure - continued

Add correction vectors from matrix sol'n to $P$ and $H$ PHREG - determine thermodynamic region for a block PHASECHG - check for phase changes

PRPTY - calculate fluid properties and derivatives AVGPRPTY - calculate interblock fluid properties WELLALLO - calculate source/sink information UPSTRE - determine upstream blocks STORATIV - calculate mass and energy storativities RESID - calculate residual mass and energy fluxes GOVEQN - sum finite-difference contributions to governing equations

check for convergence; if criteria are not met either continue NRIs or reduce time step and repeat

MAXCHG - determine maximum change in pressure, enthalpy, and saturation

End of Newton-Raphson iteration*

BALNCE - determine global mass and energy balance GOVEQN - sum finite-difference contributions If it is time to print results, then VELOCTY - calculate fluid-velocity vectors OUTPLOT - write file for input to plotting program PDATA - print results

WR - help print arrays

GOVEQN - sum finite-difference contributions

PICKUP - write pickup file for restarting jobs

GFILES - open output file

If it is time to read in new input data, then

SINK - read source/sink input data

PRINTOPT - read user-defined print options

$\mathrm{READ} \quad$ - read input arrays

$\mathrm{RD} \quad$ - help read input arrays

WR - print input arrays

GFILES - open output file

TCALC - calculate permeability and conductivity terms

PHREG - determine thermodynamic region for a block

PRPTY - calculate fluid properties and derivatives

AVGPRPTY - calculate interblock fluid properties

WELLALLO - calculate source/sink distribution and derivatives

UPSTRE - determine upstream blocks

STORATIV - calculate initial mass and energy storativities

Determine appropriate length for new time step

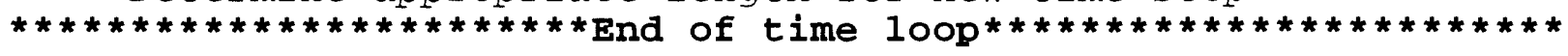

ENDSUMRY - list summary information about computer job 
Table 2. HYDROTHERM subroutines and functions for calculating fluid properties and relative permeabilities and defining the two-phase region

tblookup - determines which subset of the lookup table to call for a given pressure (P) - enthalpy (h) pair.

The limits of the sub-tables are:

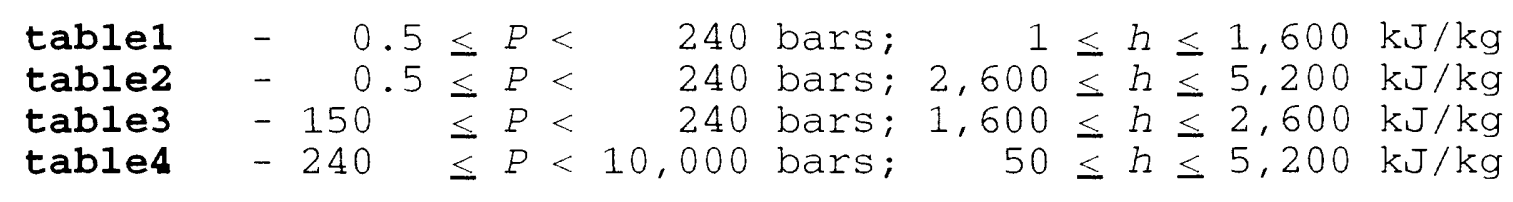

bndyro - calculates the properties of water at $0^{\circ} \mathrm{C}$ for pressures between 240 and 10,000 bars. Used in conjunction with tables to limit the lookup table to temperatures $>0^{\circ} \mathrm{C}$

The lookup table does not provide data for the two-phase region. The following three subroutines provide data on the properties of saturated liquid water and steam. For a given pressure, these subroutines return:

phsbndy1 - enthalpy

phsbndy2 - enthalpy, density, viscosity, and temperature, and the derivatives of density, viscosity, and temperature with respect to pressure and enthalpy for the onephase side of the saturation curves

phsbndy3 - enthalpy, density, viscosity, and temperature, and their derivatives with respect to pressure along the saturation curves

spline42 - interpolates fluid-property values for pressureenthalpy pairs that plot between the saturation curves and the gridded data in the lookup table.

Interpolation is done via a cubic spline, using values from phsbndy2 and the nearest point on the lookup table

tempress - uses the steam tables (Haar and others, 1984) to calculate the initial enthalpy when the user specifies initial energy content in terms of temperature

relperm - determines the relative permeabilities to liquid water and steam, and their derivatives with respect to pressure and enthalpy 
Table 3. Auxiliary files used in HYDROTHERM

These files are accessed from various subroutines via "include" statements

common.stm - common-block statements used by most of the subroutines listed in table 1

common.rdw - common-block statements used by the "read and write" subroutines ( $R D$, WR)

common.sol - common-block statements used in subroutines that set up and solve the matrix equations (FORMEQ, SOLVE, SSOR)

common.tbs - common-block statements used by the lookup table and spline routines for the properties of water (most of the subroutines listed in table 2)

hydrthrm.par-contains parameter statements that dimension arrays used in HYDROTHERM

hydrthrm.ftn-contains unit-conversion functions 
features and the modified convergence criteria. We have also standardized variable names throughout the program (see attachment $A)$ and minimized the number of variables passed between subroutines. All of these changes should facilitate future enhancements of the program. 


\section{PRACTICAL CONSIDERATIONS FOR MODEL APPLICATION}

This section summarizes the practical information needed to apply HYDROTHERM. It begins with a discussion of initial and boundary conditions, describes problem-size limitations and discretization strategies, and includes instructions on installing, compiling, and running the model. The section concludes with detailed descriptions of the input and output files.

\section{Initial conditions}

Initial conditions are the values of the dependent variables, pressure and enthalpy, at the initial problem time. Model runs may be initialized as new runs or as continuations of previous runs.

For new runs, the user may specify initial energy conditions in terms of temperature rather than enthalpy. The program will then calculate initial enthalpies on the basis of pressures and temperatures. If so desired, the program can also calculate an initial hydrostatic pressure distribution on the basis of pressures along the top of the problem domain and the initial values for temperature/enthalpy.

For continuation runs, the user retrieves pressure and enthalpy values at the end of the previous run from the output file "HTpickup_(plus user-supplied suffix)". This file also contains the simulation time at the end of the previous run, and the input parameter "tmstr" for the continuation run should be set to this value. If a non-zero rock compressibility has been specified (that is, input parameter "beta" > 0), then the continuation run requires data on initial and final porosity from the previous run. To access initial porosity values from the previous run, the user needs only to supply the name of the file 
containing these data, which is usually "HTinitphi_(plus usersupplied suffix)". Values for final porosity from the previous run are in the output file "HTpickup_(plus user-supplied suffix)", and must be used as initial porosity values for the continuation run.

Initializing new runs in the two-phase region can be problematic because, for a given pressure, very small differences in enthalpy or temperature can cause large differences in volumetric liquid saturation and thus relative permeabilities. It is difficult for the user to specify pressure/enthalpy values that will give rise to stable distributions of saturation and relative permeability. Continuation runs do not encounter this problem because the model-generated pressure, enthalpy, saturation, and relative-permeability values are internally consistent. It is often adviseable to initialize new runs such that nearly all active finite-difference blocks lie in singlephase regions, even if the boundary conditions and source/sink distribution are expected to create two-phase zones. Simulations can be carried out to a quasi-steady "natural state" in order to obtain reasonable initial conditions for analyses of geothermal development.

\section{Boundary conditions}

In addition to the initial conditions, the user must specify conditions at the boundaries of the system under investigation. The choice of appropriate boundaries is extremely important, as it often controls later-time solutions. The most common boundary conditions are (1) constant pressure/enthalpy and (2) controlled flux.

In vertical sections and three-dimensional models the top boundary, which often represents the land surface (or water table), is commonly treated as a constant pressure/enthalpy boundary. The pressure is typically specified as one atmosphere 
and, if topographic relief is significant, the specified enthalpy might vary with elevation, perhaps according to an adiabatic lapse rate. Hydrostatic lateral boundaries are commonly prescribed by column(s) of constant pressure/enthalpy blocks, with enthalpy varying according to a geothermal gradient. Such boundaries are typically placed at a large distance from the area of interest, so as to minimize their effect on the solution.

For regional and subregional flow problems, the lateral and bottom boundaries are commonly treated as controlled-flux boundaries. The lateral boundaries might be associated with topographic divides that can be treated as no-flow (symmetry) boundaries or, for a problem involving cooling of a regularly shaped pluton, symmetry might be invoked on a plane through the center of the pluton. The most common lower boundary for largescale flow problems is one with no fluid flow and specified heat flow. The lack of fluid input is justified by the assumption of low permeability at midcrustal depths, and an appropriate heat input can be estimated on the basis of regional heat flow. Within the HYDROTHERM model, conductive heat flux along the base of the grid ("cond") is actually implemented as a source within the lowermost active blocks. It is usually much easier to estimate fluxes at the lower boundary than it is to estimate appropriate pressures and enthalpies, and constant pressure/enthalpy lower boundaries tend to have an undesireable controlling effect on the solution.

To set up constant-value and no-flow boundaries, HYDROTHERM uses the following convention for indexing blocks and allowing flow between blocks. In the input file, the user labels active blocks with sequential positive-integer values, constant blocks with -1 , and inactive blocks with 0 . Interfaces between active (integer $\geq 1$ ) and inactive (0) blocks are no-flow boundaries, as are the sides of active blocks that lie on the grid boundaries. Flow between constant blocks and adjacent active blocks varies 
with the pressure/enthalpy conditions in the active block. Twodimensional examples showing grid index numbers and associated boundary conditions are shown in figure 2 .

\section{Problem size}

The maximum feasible problem size is a function of the memory and/or disk space available on the computer. In practice, the time required to run large problems may prove to be a more restrictive limit. HYDROTHERM can be set up for any dimensions by changing the parameter statements in the file "hydrthrm.par". Instructions for setting the parameter values are included in "hydrthrm.par". The program must be recompiled after the maximum dimensions are reset. The copy of "hydrthrm.par" distributed with the program is designed to handle a $50 \times 5 \times 50(x \times y \times z)$ block (maximum) problem.

\section{Notes on discretization}

The $x^{-}, y^{-}$, and $z$-dimensions of finite-difference blocks may vary along the axis of that dimension, but are held constant in the other two dimensions. For example, in a two-dimensional vertical $(x-z)$ slice, each row may have a different $z$-dimension, but along any row, the $z$-dimension may not vary as a function of $x$. In order to improve the definition of the solution, it is often desireable to set up a model with a higher density of columns $(x)$, rows $(z)$ and slices $(y)$ in areas of interest and (or) areas where large gradients in pressure and enthalpy are expected, in order to improve definition of the solution. Conversely, using coarser grid spacing near poorly known boundaries helps to minimize their influence on later-time solutions. A useful and fairly conservative rule of thumb is that boundary influence will be negligible when the distance to the boundary is ten times greater than the width of the area of interest. 


\section{A. VERTICAL SECTION}

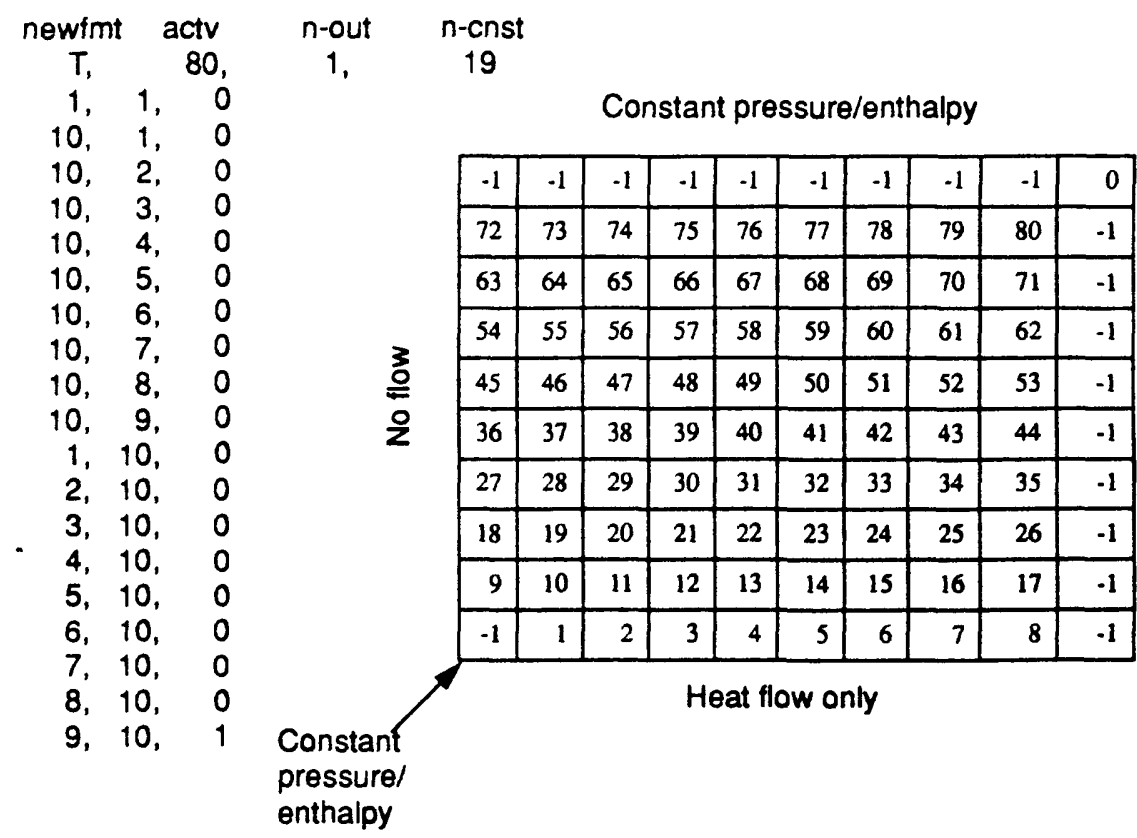

\section{B. VERTICAL SECTION WITH TOPOGRAPHY}

$\begin{array}{ccc}\text { newfmt } & \text { n-actv } \\ \text { T, } & \mathbf{5 8 ,} \\ 1, & 9, & 0 \\ 2, & 9, & 1 \\ 3, & 8, & 1 \\ 4, & 7, & 0 \\ 5, & 7, & 0 \\ 6, & 7, & 1 \\ 7, & 6, & 0 \\ 8, & 6, & 1 \\ 9, & 5, & 1 \\ 10, & 4, & 1\end{array}$

\begin{tabular}{|c|c|c|c|c|c|c|c|c|c|c|}
\hline \multirow{10}{*}{$\begin{array}{l}\frac{3}{0} \\
\text { 을 } \\
\text { 2 }\end{array}$} & \multicolumn{10}{|c|}{ Constant pressure/enthalpy } \\
\hline & -1 & -1 & 0 & 0 & $\mathbf{0}$ & $\mathbf{0}$ & 0 & 0 & 0 & \\
\hline & 57 & 58 & -1 & 0 & 0 & 0 & $\mathbf{0}$ & 0 & 0 & 0 \\
\hline & 54 & 55 & 56 & -1 & -1 & -1 & 0 & 0 & 0 & 0 \\
\hline & 48 & 49 & 50 & 51 & 52 & 53 & -1 & -1 & 0 & 0 \\
\hline & 40 & 41 & 42 & 43 & 44 & 45 & 46 & 47 & -1 & 0 \\
\hline & 31 & 32 & 33 & 34 & 35 & 36 & 37 & 38 & 39 & -1 \\
\hline & 21 & 22 & 23 & 24 & 25 & 26 & 27 & 28 & 29 & 30 \\
\hline & 11 & 12 & 13 & 14 & 15 & 16 & 17 & 18 & 19 & 20 \\
\hline & 1 & 2 & 3 & 4 & 5 & 6 & 7 & 8 & 9 & 10 \\
\hline
\end{tabular}

Heat flow only

Figure 2. Example finite-difference grids for simulating vertical sections, showing finite-difference-block index numbers, resulting boundary conditions, and "newfmt" input format for finite-difference-block indexing. 
Ingebritsen (1986) used the 1982 version of the code, GEOTHER, to simulate the evolution of vapor-dominated zones and noted (p. 166-170) some space-discretization effects. These effects included unrealistic fluctuations in mass and heat discharge from an evolving vapor-dominated zone and difficulty in defining the "upstream" flow direction at the top of the vapordominated zone, an area where the pressure gradient changes radically. When strong discretization effects are suspected, problems should be rerun with a revised, refined grid. The orientation of the finite-difference grid can also have a major influence on simulations of vapor-dominated zones (Pruess, 1991b).

\section{Convergence and time-step control}

HYDROTHERM allows the user to specify convergence criteria for the Newton-Raphson iterations, as well as several other factors that can act to control the size of the time step. The choice of values affects the time required to run a problem, the stability of the algorithm, and the accuracy of the solution.

Convergence to a solution is achieved when every block satisfies user-specified mass- and energy-balance criteria. These criteria define the acceptable level of error in the solution of the two governing equations (eqns. 1 and 2), each of which should theoretically sum to zero. The residual is the difference from zero. The convergence criteria for HYDROTHERM specify the maximum residual mass flux per unit volume $\left(\mathrm{g} / \mathrm{sec} / \mathrm{cm}^{3}\right)$ and the maximum residual energy flux per unit volume (ergs $/ \mathrm{sec} / \mathrm{cm}^{3}$ ). The value for the residual energy flux should generally be about 10 orders of magnitude greater than that for residual mass flux, because the enthalpy of liquid water at $225^{\circ} \mathrm{C}$ is approximately $10^{10} \mathrm{ergs} / \mathrm{g}$. We have found that mass and energy residuals in the ranges of $10^{-14}-10^{-12}$ and $10^{-4}-10^{-2}$, respectively, give good results. Looser criteria (values up to $10^{-10}$ and $10^{\circ}$, respectively) may produce adequate results and can 
significantly reduce computational time. In some cases, however, the looser criteria lead to poor results. In practice, it is sometimes useful to start new problems with loose criteria and recheck the solution with tighter criteria.

A total of nine factors control the size of a new time step. Table 4 lists each factor, along with suggested values, and also lists the eight factors that can cause the program to discard a solution and re-enter the Newton-Raphson loop with a smaller time step.

HYDROTHERM sets the time-step length by determining which factor is likely to be most restrictive and setting the size for the next time step accordingly. Maximum changes in saturation, pressure, and enthalpy are estimated on the basis of the previous time step. As with the convergence criteria, it is sometimes convenient to start a new problem with loose limits (larger values) that allow the program to run relatively fast, and then recheck solutions with tighter limits.

HYDROTHERM will decrease the size of a time step if there is a problem with convergence to a solution, or if other time-stepcontrol factors exceed the user-specified limits. Six of the eight reasons for decreasing time-step size depend on userspecified criteria (Table 4). For example, the program will stop if a particular time step is cut more than a user-specified number of times ("n-tmcut") or if the time step becomes smaller than "deltmin". The program will reduce the time step automatically if the pressure or enthalpy of a block exceeds the limits of the lookup table, or if the Newton-Raphson iterations do not appear to be converging toward a solution: if the mass and energy residuals for the current iteration are both larger than those from the second-previous iteration, the program stops iterating and re-enters the Newton-Raphson loop with a smaller time step. 
Table 4. Factors that control the time step in HYDROTHERM

The one-letter abbreviations are used in output file HTouto when the short report ("long" = 0) is selected. In longer reports ("long" = 1 or 2), the controlling factor is written out

\section{Factor}

Maximum water saturation change Maximum percent pressure change Maximum percent enthalpy change Maximum increase in time step size Input value for initial step Time to read new input data Time to plot or print data Reset to value before printing

Reset to value before reading new data $R$
W

$\mathrm{P}$

$\mathrm{H}$

$\mathrm{M}$

I

D

0

R

\section{Abbrev. Suggested range}

$$
\begin{aligned}
0.02 & -0.10 \\
10 & -30 \\
4 & -10 \\
1.1 & -2.0
\end{aligned}
$$

highly variable program controlled program controlled program controlled program controlled

\section{Reasons for cutting the time step}

Saturation change exceeded maximum allowed

Pressure change exceeded maximum allowed

Enthalpy change exceeded maximum allowed

Multiple blocks changing phase in a Newton-Raphson iteration Multiple blocks changing phase in a time step Maximum Newton-Raphson iterations reached without convergence Newton-Raphson iterations not converging

Pressure or enthalpy exceeds limits of lookup table 


\section{Installing and compiling the program}

HYDROTHERM is written in an extended, UNIX-compatible version of Fortran. We did most of the development and testing on a Silicon Graphics Iris Indigo work station. We also compiled and ran this version on a Data General work station. Hayba (1993) designed and ran an earlier version on an Alliant supercomputer, and others used that same version on Sun, Prime, and Hewlett-Packard computers. Some of the program revisions take advantage of the parallel-and vector-processing capabilities of the Alliant supercomputer, but these modifications do not have an adverse affect on non-parallel or non-vectorizing machines.

To install HYDROTHERM, some code modification may be necessary, especially on machines than do not use the UNIX operating system, or for Fortran compilers that do not support some of the Fortran extensions. The required changes are listed below. Revision dates should be recorded in the comment statement at the head of each file. We request that users advise the senior author of any additional machine- or Fortran-versionspecific problems.

Users must first copy all of the files listed in table 5 to an appropriate sub-directory in their file-space, or to a publicly accessible directory such as "/usr/1ocal/bin" on UNIX machines. When copying the files with the "ftp" utility, it is necessary to use the ascii mode for all but the six files designated as binary in table 5. The user must edit "gfiles.f" (1ines 120-131), and make sure that the directory names for units 21 to 26 are correct. As noted above under "Problem size", the user may also reset the maximum number of blocks in the $\mathrm{x}^{-}, \mathrm{y}^{-}$, and $z$-dimensions, using the parameter statements in "hydrthrm. par".

Green Hill Fortran does not support the "fdate" command that 
Table 5. Complete list of HYDROTHERM program files

\section{Installation files}

Makefile hydrthrm.ver

\section{Fortran source-code files}

$\begin{array}{lllll}\text { avgprpty.f } & \text { main.f } & \text { press.f } & \text { spline42.f } & \text { timecntl.f } \\ \text { balnce.f } & \text { maxchg.f } & \text { printopt.f } & \text { ssor.f } & \text { upstre.f } \\ \text { bndyTo.f } & \text { outplot.f } & \text { prpty.f } & \text { storativ.f } & \text { velocty.f } \\ \text { endsumry.f } & \text { pdata.f } & \text { rd.f } & \text { tablel.f } & \text { wellallo.f } \\ \text { enthtemp.f } & \text { phasechg.f } & \text { read.f } & \text { table2.f } & \text { wr.f } \\ \text { formeq.f } & \text { phreg.f } & \text { relperm.f } & \text { table3.f } & \text { wrnxx.f } \\ \text { gdata.f } & \text { phsbndyl.f } & \text { reset.f } & \text { table4.f } & \text { wrnzz.f } \\ \text { gfiles.f } & \text { phsbndy2.f } & \text { resid.f } & \text { tblookup.f } & \\ \text { goveqn.f } & \text { phsbndy3.f } & \text { sink.f } & \text { tcalc.f } & \\ \text { gtables.f } & \text { pickup.f } & \text { solve.f } & \text { tempress.f } & \end{array}$

Common-block and related parameter and function files common.rdw common.sol common.stm common.tbs hydrthrm. ftn hydrthrm.par

\section{Binary files for lookup tables and cubic splines}

proptable1 proptable2 proptable3 proptable4 splnto splnws 
HYDROTHERM uses to record the time and date of the computer run. Comment statements near line 200 in "gdata.f" indicate the necessary modifications for machines that use this compiler.

Non-UNIX machines, such as Prime computers, require more extensive modifications. In addition to the "fdate" command, the user must modify or "comment out" the "maxval" command in "pdata.f" and the "etime" command in "endsumry.f". Three lines in "gfiles.f" must also be bypassed, as indicated by comment statements near the head of the file.

To compile HYDROTHERM on UNIX machines, the user must edit "Makefile" and set the appropriate machine-specific options. Before running "make", the user must also create a subdirectory named "O" to store the object files. Upon successful compilation, the "Makefile" will check the revision dates recorded in the comment statements at the head of each file, and report which files have been modified. For any file that has been changed, the "Makefile" will report the different dates as well as the following line

$\star \star \star$ Error code 1 (ignored),

which can be ignored. The file "hydrthrm.ver" contains the revision dates for each file, and "Oldver", which the "Makefile" creates after the first compilation, contains the revision dates for the previous compilation.

\section{Running the program}

When the user has successfully compiled the program, the computer produces an executable version of HYDROTHERM named "HT". The command line to run HYDROTHERM on UNIX machines is

HT input_filename suffix 
where "input_filename" is the name of the file that contains the input file (discussed in the next section), and "suffix" is a user-specified suffix to be appended to the name of each output file created by this run. The default input file is "HTin". A suffix may not be specified unless the user also specifies the input file.

For non-UNIX machines, the command line is simply "HT". The input file must be in "HTin", and no suffix will be appended to the output files.

\section{Input file}

The input file for HYDROTHERM consists of a template alternating with input values. This template simplifies entry of input parameters by providing a cryptic user guide, thereby minimizing the need to refer to this report when developing input files for specific problems. Input files for each of five example problems are included as attachments to this report. Specific input options are discussed in greater detail below, and some recommended or default parameter values are given in brackets .

\section{TITLE -}

Two lines are provided for the title; 72 spaces may be used on each line. The first line must begin with the letters "TITLE", and the second line with the letters "2FORM". The program checks for these key words to ensure that the proper input format is being used

\section{DIMENSIONS -}

$\mathbf{n x}$ number of blocks in the $\mathbf{x}$ direction, or in $r$ direction for radially symmetric problems

ny number of blocks in the $y$ direction; must be 1 for radial problems

$\mathbf{n z}$ number of blocks in the $z$ direction 
bndwth maximum width of the matrix. Program will calculate this value if "bndwth" is set to zero [0]

tmstr simulation time at the beginning of the run. For new runs this value is usually 0 .; for continuation runs it is usually obtained from the output file HTpickup. Note that parameter units must agree with "iyr"

iyr $T$ indicates that times are input to the program in years; $F$ indicates that times are input in seconds

TIME STEP -

The program calculates time steps on the basis of a number of user-specified parameters, including the time-step increase factor, maximum allowable changes in pressure, enthalpy, and saturation, and print frequency

tstepmx maximum number of time steps for simulation run

tmincr maximum time-step increase factor [1.3]

\% Pchg maximum percentage change in pressure that any block can experience in a given time step [10.0]

\%chg maximum percentage change in enthalpy that any block can experience in a given time step [5.0 to 7.0]

wsatn maximum change in water saturation that any block can experience in a given time step [0.03]

relax maximum number of blocks through which a fluid particle can flow in a given time step (Note: This option is not yet implemented)

deltmin minimum time step; the run is stopped if the time step is cut to a value less than "deltmin", except on the first time step. Note that parameter units must agree with "iyr"

n-tmcut maximum number of times that a particular time step can be cut and repeated before the run is terminated [10]

\section{N-R ITERATION -}

Time steps are halved and repeated if user-specified convergence criteria are not met in "itermx" interations 
itermx maximum number of Newton-Raphson iterations allowed per time step. If the convergence criteria are not satisfied in "itermx" iterations, the time step is halved and tried again [5 to 12 ]

resmas the Newton-Raphson mass-balance convergence criterion: maximum residual mass flux per unit volume $\left(\mathrm{g} / \mathrm{sec} / \mathrm{cm}^{3}\right)$ [1.0D-14 to $1.0 \mathrm{D}-12]$

reseng maximum residual energy flux per unit volume $\left(\mathrm{erg} / \mathrm{sec} / \mathrm{cm}^{3}\right)$. This value should generally be about ten orders orders of magnitude greater than "resmas"; the enthalpy of liquid water at $225^{\circ} \mathrm{C}$ is approximately $1.0 \mathrm{E} 10 \mathrm{ergs} / \mathrm{gm}) \quad$ [1.0D-4 to $1.0 \mathrm{D}-2]$

nphasechg - the maximum number of blocks that can change phase in one Newton-Raphson iteration $[\leq 10]$

\section{3-D SSOR CONTROL -}

These three variables apply to 3-dimensional problems only; any value can be input for 1- or 2-dimensional simulations

ssormx maximum number of slice-successive overrelaxation iterations per time step [3 to 5]

tol tolerance on the ssor iterations [0.05]

ssorwo the ssor relaxation factor [1.0]

\section{WEIGHTING \& AVERAGING -}

ioptupst $T$ indicates upstream weighting for enthalpy and relative permeability; $F$ indicates midpoint weighting

potdif difference in potential between two blocks above which full upstream weighting (if selected) is used; below this value, a weighted average of upstream and downstream values is used. This feature is intended to facilitate smooth changes in flow direction [0.0002]

theta time weighting value; 1 indicates fully implicit, 0 indicates fully explicit (Note: This option is not yet implemented) [1.0 must be used] 
kodrp 0 specifies linear relative permeability functions:

$$
\begin{aligned}
& k_{r W}=S S \text { and } \\
& k_{r S}=1-k_{r W} ;
\end{aligned}
$$

1 specifies Corey-type functions (Corey, 1957):

$$
\begin{aligned}
& k_{r w}=S S^{4} \text { and } \\
& k_{r S}=\left(1.0-S S^{2}\right) \times(1.0-S S)^{2} ; \text { and }
\end{aligned}
$$

2 specifies "fracture-flow" functions (Sorey and others, 1980):

$$
\begin{aligned}
& k_{r W}=S S^{4} \text { and } \\
& k_{r S}=1-k_{r W^{\prime}} \\
& \text { where } S S=\left(S_{W}-S_{W r}\right) /\left[\left(1.0-S_{W r}\right)-S_{S r}\right]
\end{aligned}
$$

wsatn residual liquid water saturation $\left(S_{W r}\right.$ above)

ssatn residual steam saturation $\left(S_{S r}\right.$ above)

\section{ROCK PROPERTIES -}

heatcpty rock enthalpy derivative with respect to temperature (that is, specific heat of rock) (erg/g/K)

rxden rock density $\left(\mathrm{g} / \mathrm{cm}^{3}\right)$

rxcmprss - rock compressibility ( $\mathrm{cm}^{2} /$ dyne);

if "rxcmprss" is greater than zero, a file containing the initial pressure and porosity values is created and given the name HTinitphi (plus suffix, if used). This file is needed for restarting runs (see "initphi" below) grav gravitational constant $\left(\mathrm{cm} / \mathrm{sec}^{2}\right)[981$. , or 0 for two-dimensional areal models]

initphi $T$ indicates that initial (original) pressure and porosity values are to be read in; the name of the file containing these values must be given on the following line. $F$ indicates that no initial (original) porosity and pressure values are to be input. Note that this option is only used for continuation runs in which the rock compressibility is greater than zero. For these runs the initial pressure and porosity values (that is, the values input for the original run) are required in 
order to correctly calculate changes in storativity and the new porosity values

\section{RADIAI COORDINATES -}

irad $T$ indicates that radial coordinates are to be used; $F$ indicates that Cartesian coordinates are to be used

wellrad well radius $(\mathrm{cm})$

radiusmax - maximum reservoir radius (cm)

\section{PRINT/PIOT -}

prfreq frequency for printing and plotting results (in years or seconds, depending on "iyr"). If a negative integer value is input, the results will be output every step, beginning with the step number specified by the absolute value of the integer. Note that using a negative integer can produce very large output files; this option is included as a debugging tool

plot 0 indicates that a plot-file should not be produced; 1 indicates that a formatted plot-file should be sent to HTpltout; 2 sends an unformatted plot-file to HTpltout. Note that the outplot.f subroutine is not included in most releases of the code

wide 0 indicates that an 80-column-wide format should be used for output files (other than HTplotout); 1 indicates that a 132-column-wide format should be used

long 0 indicates that limited output should be written to HTouto; 1 indicates moderate output to HTouto; 2 indicates long output to HTouto

v-avg 0 indicates that vertically averaged values should not be written to HTout1; 1 indicates that HTout 1 should include vertically averaged values for pressure, enthalpy, temperature, and (or) water saturation (depending on which arrays are selected for printing; see PRINT 1 below)

ssflx 0 indicates that source/sink and constant-block flux 
data should not be written to HTout5; 1 indicates that

this information should be written to HTout 5

pnodes the number of specific blocks for which pressure, enthalpy, temperature, and saturation data are output to file HTout4. The format of this file facilites input to a spreadsheet or graphics program for plotting. This is useful for tracing conditions in a few key blocks throughout a run. The $i, j, k$ indices for each of the "pnodes" must follow on successive lines

The following three lines include on/off switches for writing arrays to specific files

$$
0=\text { not selected, } 1=\text { selected to be printed }
$$

\section{PRINT 1 -}

Environmental characteristics - output to file HTout 1

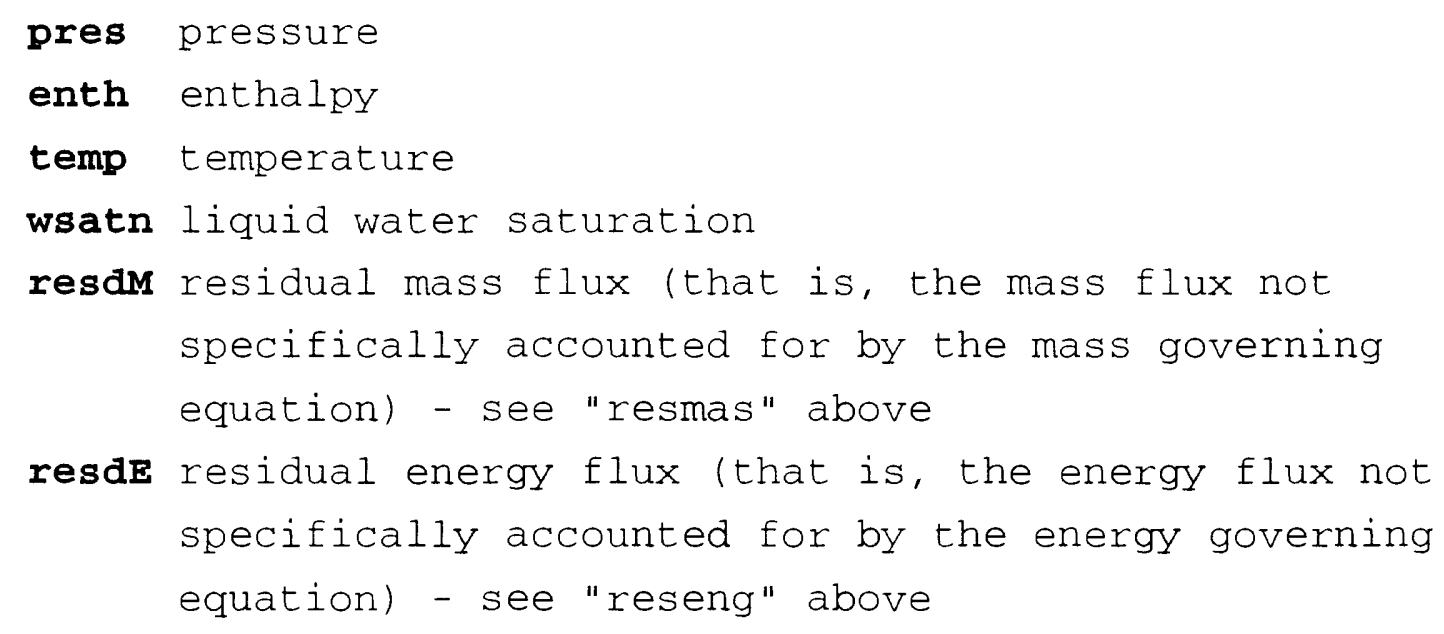

resde residual energy flux (that is, the energy flux not specifically accounted for by the energy governing equation) - see "reseng" above

\section{PRINT 2 -}

Liquid water characteristics - output to file HTout2

wden density

wris viscosity

wxvel velocity of liquid water in the $\mathrm{x}$ direction

wrvel velocity of liquid water in the $y$ direction (not yet implemented)

wzvel velocity of liquid water in the $z$ direction wpotnl liquid water potential 


\section{PRINT 3 -}

Steam characteristics - output to file HTout3

sden density

svis viscosity

sxvel velocity of steam in the $x$ direction

syvel velocity of steam in the $y$ direction (not yet implemented)

szvel velocity of steam in the $z$ direction

spotnl steam potential

\section{SLICE \# -}

Finite-difference-block indexing; read in a series of lines for each $x z$ slice of the model. See figure 2 for examples

newfmt $T$ indicates that the sequence numbering of blocks in this slice should be done semi-automatically. If this option is chosen, read a separate line for each constant-value pressure/enthalpy block in this slice. Each line must include $i, k, n$ where $i$ and $k$ are indices of the constant-value block and $n=-1,0$, or 1 . The $n$ values are used to help describe irregular geometric boundaries; $n=-1$ indicates that blocks in the $-x$ direction from the $i, k$ block are outside the problem domain, $n=1$ indicates that blocks in the $+x$ direction are outside the problem domain, and $n=0$ indicates that blocks in both the $-x$ and $+x$ directions are within the problem domain.

$F$ indicates that the sequence numbering of blocks in this slice will be done manually. If this option is chosen and "nz">"nx", read a line(s) for each row of this slice beginning with the bottom row of the model. Begin sequence numbers with 1 and increment by 1 for each active block in the slice, working upward row by row; input 0 for blocks outside the problem domain and -1 for constant-value pressure/enthalpy blocks. This is the input method used with the 1982 version, GEOTHER. 
If "nx">"nz", the procedure is similar, except that the sequence numbers must be incremented along columns, rather than rows

n-actv number of active blocks in this slice

n-out number of blocks in this slice that are outside the problem domain

n-const number of constant-value blocks in this slice; if this value is greater than zero and "newfmt"=T then "n-const" lines listing $i, k, n$ values must follow this line

\section{PARAMETER -}

These lines define the finite-difference grid and assign initial values to the medium properties. Eleven parameters are required and are input in the order listed below. The first line for each parameter identifies the array to be input, the second line specifies the input format, and the following line(s) contain the data. Blocks that are outside the problem domain require dumm values

The order of data input for the grid spacing is:

$x$-spacing - left to right;

$y$-spacing - front to back; and

$z$-spacing - bottom to top

Basal heat flow is input from left to right along each row $(x)$ from the front row to the back ( $y$ )

The order of data input for all of the other parameters is slice by slice $(x, z)$, starting with the bottom left block and working up each slice row by row

\section{Array Identification Lines (in the order to be input)}

The identification code must be in capitals and begin in the first column of the line

XSPA $x$ (or $r$ ) spacing of blocks (cm). For Cartesian 
coordinates, input the $x$-dimension of each block; for radial coordinates, input the radius of each block from the center of the well. (For radial coordinates, automatic spacing of blocks is recommended; see CALC below)

YSPA $Y$ spacing of blocks (cm)

ZSPA $z$ spacing of blocks (cm)

PORO porosity (unitless)

XPER $x$-direction permeability $\left(\mathrm{cm}^{2}\right)$

YPER $Y$-direction permeability $\left(\mathrm{cm}^{2}\right)$

ZPER $z$-direction permeability $\left(\mathrm{cm}^{2}\right)$

THER thermal conductivity (ergs/ $\mathrm{sec} / \mathrm{cm} / \mathrm{K}$ )

cond conductive heat flow along base of model $\left(\mathrm{mW} / \mathrm{m}^{2}\right.$, ergs/s/ $\mathrm{Cm}^{2}$ )

TEMP or ENTH - either temperature $\left({ }^{\circ} \mathrm{C}\right)$ or enthalpy (ergs/g) may be input; if temperature data is input, enthalpy will be calculated from the temperature and pressure data. Lookup-table limits are $0.01 \times 10^{9}<h<52.0 \times 10^{9}$ ergs/g.

PRES pressure (dynes/ $\left.\mathrm{cm}^{2}\right)$. Lookup-table limits are $0.5 \times 10^{6}$ $<P<1,200 \times 10^{6}$ dynes $/ \mathrm{cm}^{2}$

\section{Format lines -}

A format line follows each "array identification line". The specified format or code (FREE, CONS, CALC, etc.) must be in capital letters and begin in the first column of the line

Specified format - if a fixed format is required, such as 8F10.3, read (8F10.3). In this case the format line must consist of a list of format specifications enclosed in parentheses, but without the word FORMAT or a statement label. This is known as "execution time format specification". The other format-line options are:

FREE indicates free formatting

cons indicates a constant-value array; only one free-format 
value is listed on the next line

Rowc indicates constant values within each row; input one value for each row in the problem domain, starting with the bottom row in the first slice

colc indicates constant values within each column; input one value for each column in the problem domain, starting with the first column in the the first slice

NODE indicates that values are to be input for specific blocks. This format provides an easy way to change parameter values at a few blocks during a simulation; it is not intended to be used for specifying initial conditions. On lines following "NODE", input $i, j, k$ and a new parameter value, where $i, j, k$ are block indices. There is no limit to the number of blocks that may be assigned new values using this format, but following the last new value a line reading "0,0,0,0" must be input to signal the end of a NODE sequence. Parameter values for blocks not individualy specified by NODE are unchanged

CALC Indicates that input values for the array are to be calculated by the program. This option presently applies to only five arrays:

XSPA, for calculating the $x$ spacing of blocks when radial coordinates ("irad"=T) are used. On the following line, the radius $(\mathrm{cm})$ of the first block from the center of the well is specified

YPER, for calculating the distribution of $y$-direction permeability as a function of the $x$ permeability. On the following line, a multiplication factor is specified. For example, a value of 10.0 specifies that the $y$ permeability is an order of magnitude greater than the $x$ permeability

ZPER, for calculating $z$ permeability as a multiple of $x$ permeability in a similar fashion

COND, for calculating a linear distribution of basal conductive heat flux for each slice, from block $i=1$ to 
block $i=$ "nx". On the following line, conductive heat fluxes at blocks $i=1$ and $i=$ "nx" $\left(\mathrm{mW} / \mathrm{m}^{2}\right)$ are specified in free format

PRES, for calculating an initial hydrostatic pressure distribution. On the following line the pressure at the top of the problem domain is specified

\section{TIME PERIOD \# -}

TIME PERIOD lines specify the initial time step and number of source and sinks and, more importantly, can also be used to change conditions at any time during a simulation run. Contributions from sources and sinks may be varied, print options modified, and several of the PARAMETERS changed by using a TIME PERIOD line and any additional required lines. There is no limit to the number of TIME PERIOD lines, but they must be input in chronological order. The last line in the file should have "tchg" = -1 to terminate the run

tchg time (in years or seconds, depending on "iyr") at which this period will end and a new TIME PERIOD line will be read. A value of -1 will terminate the run and should appear on the last line in the file

delt initial time step (in years or seconds, depending on "iyr"). After the first time period, the time step will be calculated automatically if "delt" is specified as -1

nsrce number of SOURCE/SINK lines to follow; - 1 indicates no change in the SOURCE/SINK lines from the previous time period

ichgpr $T$ indicates that any or all of the previously specified print/plot options should be changed and requires the PRINT/PLOT and PRINT lines described above to follow. F indicates that the print/plot options are not to be changed

nparms number of PARAMETERS to be redefined. At any time during the run, porosity, $x^{-}, y^{-}$, and $z$-permeability, 
thermal conductivity, conductive heat flux, temperature/enthalpy and (or) pressure may be modified. The required lines are identical to the PARAMETER lines described above and follow the SOURCE/SINK and PRINT lines (if any). Recall that the NODE format option provides an easy way to change the values of a few blocks

\section{SOURCE/SINK \# -}

"nsrce" of these lines must follow the TIME PERIOD line. There is a limit of one well per $i, j$ location

$i$ the $i$ index location of the well

$j$ the $j$ index location of the well

w1flx the mass flux associated with the well (g/sec)

Positive values indicate injection and negative values indicate withdrawal

wlenth enthalpy of the injection fluid (ergs/g); input 0.0 for withdrawal

n-openint - number of open intervals (blocks) contributing to or accepting mass from this well. The $k$ indices of "nopenint" blocks follow this line

\section{Output files}

HYDROTHERM produces 2-8 output files, depending on the user's choice of options. Only HTouto and HTpickup are produced for every simulation run. The amount of information included in most files is user-specified. Characteristics of the output files are summarized in table 6 .

HTouto provides the user with a reformatted copy of the input file, tracks the progress of the simulation run, and concludes with summary information about the run. Limited-, moderate- and long-output options are offered. If the user specifies a limited amount of output ("long" = 0), each time step is summarized on a single line that lists time-step length, the 
Table 6. HYDROTHERM output files

HTouto Tracks progress of a simulation run. Limited-, moderate-, and long-output options are offered

Hout 1 Contains fundamental information about the solution (Any or all of pressure, enthalpy, temperature, saturation, residual mass, residual energy)

Hout2 Liquid-water characteristics (density, viscosity, $x$ - and $z$-direction velocities, "potential")

HTout3 steam characteristics (density, viscosity, $x-$ and $z-$ direction velocities, "potential")

HTout4 Contains pressure, enthalpy, temperature, and saturation values for selected blocks

HTout5 contains fluxes for constant blocks, sources, and sinks

HTpickup-Contains continuously updated pressure/enthalpy solution that is used to initialize continuation runs

HTintiphi-Stores initial pressure and porosity values for

problems involving non-zero rock compressibility

HTplotout-Output file for plotting that is generated by subroutine outplot.f (this subroutine is not included with most releases of the code) 
factor that controlled time-step length, cumulative simulation time, and the number of Newton-Raphson iterations, time-step reductions, and phase changes. Summary information at the end of HTouto includes statistics on time-step length and the factors causing time-step reductions. The moderate-output option ("long" =1) generates much more information. Results from each NewtonRaphson iteration are summarized in terms of maximum mass and energy residuals, and the $i, j, k$ indices of the blocks with maximum residuals are listed. The individual time-step summaries are also expanded, and the overall length of HTouto is increased by a factor of 10 or more relative to the limited-output option ("long" = 0). The long-output option ("1ong" = 2) expands the summary of each Newton-Raphson iteration and also includes global mass and energy balances for each time step. It increases the overall length of HTouto by approximately a factor of five relative to the moderate-output option ("long" = 1 ).

HTouto files generated using the moderate- and long-output options can prove very helpful when the user is having difficulty running a particular problem. However, these options can easily generate an unacceptably long output file and should not be used routinely. The "long" = 2 option, in particular, should only be used for problem debugging.

Simulation-time output intervals for HTout1 through HTout5 are specified by "prfreq". HTout1 through HTout3 include parameter values for each block, and 80-or 132-column-wide format options for these files are specified by "wide".

Fundamental information about the solution is written to HTout1. This file includes any or all of pressure, enthalpy, temperature, saturation, residual mass flux, and residual energy flux. 
The HTout2 and HTout 3 files include any or all of fluid density, viscosity, $x$ - and $z$-velocities, and "potential". HTout2 includes values for liquid water and fluid above the critical pressure of 220.55 bars, and HTout3 includes steam parameters. The velocities are fluid-particle velocities, rather than Darcian velocities $\left(v_{p}=v_{D} / n\right.$, where $v_{p}$ and $v_{D}$ are fluid-particle and Darcian velocities, respectively). The "potential" is actually a pseudo-potential; flow of compressible fluid is not a potential flow, except for the special case of $\rho(P)$ (Bear, 1972, p. 159f), which does not apply to geothermal problems. Thus the differences in "potential" $\left(\Phi_{i}=P_{i}+\rho_{i} g z_{i}\right)$ between adjacent blocks do not correspond exactly to the driving forces that the model uses to calculate fluxes.

HTout 4 includes pressure, enthalpy, temperature, and saturation values at selected "pnodes" for which $i, j, k$ indices are specified. The output format is convenient for plotting. HTout5 is created if "ssflx" = 1 , and includes source/sink and constant-block fluxes.

HTpickup contains the continuously updated pressure/enthalpy solution. The information contained in HTpickup is needed to initialize continuation runs. On some systems, attempting to examine this file during a simulation run will cause the program to crash. If rock compressibility is non-zero, the initial pressure and porosity values stored in HTinitphi are also needed to initialize continuation runs.

HTpltout is an output file for plotting that is generated by the subroutine outplot.f, which is not included with most releases of the code. 


\section{EXAMPLE PROBLEMS}

Five example problems presented below serve to demonstrate the thermodynamic range and multi-dimensional performance of the HYDROTHERM model. Analytical solutions for constant-enthalpy and constant-pressure injection into a single finite-difference block can be used to check the thermodynamic performance of the model. The simulated temperature distribution for steady one-dimensional upflow can be compared with the single-phase analytical solution of Bredehoeft and Papadopolous (1965) and with a boiling pointdepth curve. A solution for radial flow to a well can also be checked semi-analytically, but we rely on previously published numerical results to check solutions for an expanding, onedimensional two-phase zone and three-dimensional flow.

\section{One-block problem}

Roberts and others (1987) used constant-enthalpy and constant-pressure single-block experiments to test their extended version of the GEOTHER (Faust and Mercer, 1982) simulator. We describe similar experiments here. Input files for constantenthalpy and constant-pressure problems are included as Attachments $B$ and $C$ to this report, respectively.

For constant-enthalpy injection (table 7, tests 1-3), volume is fixed by specifying an incompressible rock matrix. Fluid is injected into a $1 \mathrm{~m}^{3}$ block at a rate $q_{m}(M / T)$ for time $t$. The net increase in mass, $\Delta M$, is $q_{m} \times t$. The mass increase can also be calculated from the change in fluid density in the volume, $V$, as

$$
\Delta M=\left(\rho_{2}-\rho_{1}\right) V
$$

Values obtained using the two expressions for $\Delta M$ should be equivalent. 
Table 7. Results from application of HYDROTHERM to single-block injection tests

[Abbreviations: $P$, pressure; $P_{1}$ and $P_{2}$, initial and final pressure, in contant-enthalpy tests; $h$, enthalpy; $h_{1}$ and $h_{2}$ ' initial and final enthalpies, in constant-pressure Eests; $\hat{\rho}_{1}$ and $\rho_{2}$, initial and final densities; $q_{m}$ and $q_{h}$, mass and energy injection rates, respectively; ${ }^{\prime}$, time; $V$, block volume; $T_{1}$ and $T_{2}$, initial and final temperature; $M$, mass of fluid]

\section{Constant-enthalpy tests \\ ("rxcmprss" arbitrarily low)}

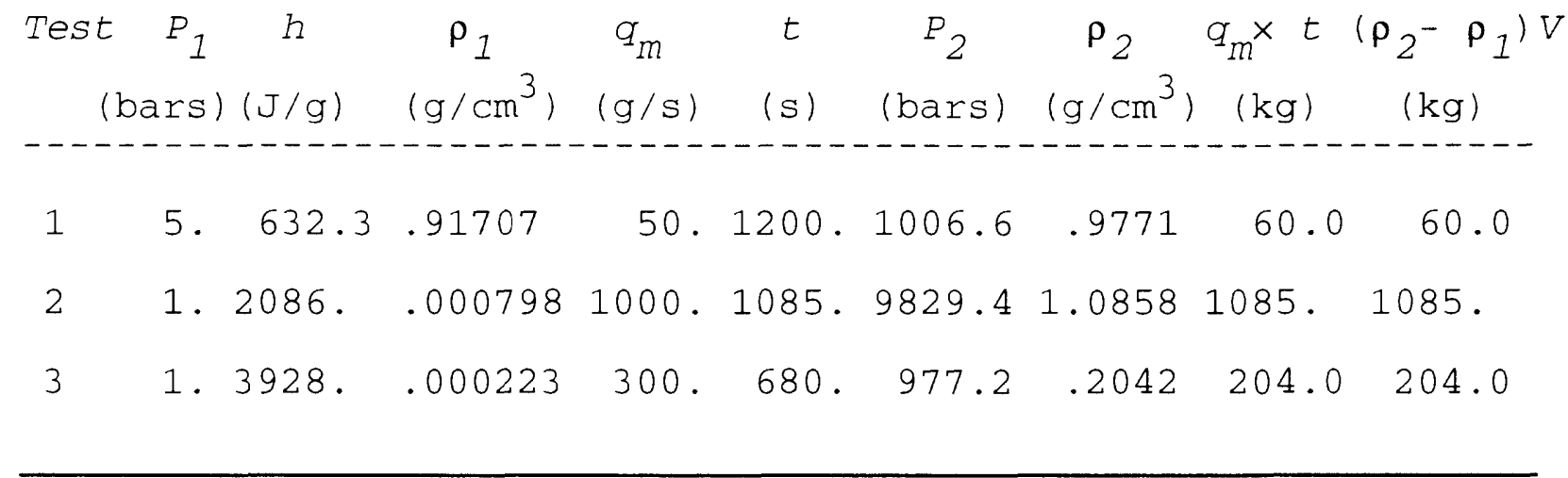

\section{Constant-pressure tests}

("rxcmprss" arbitrarily high)

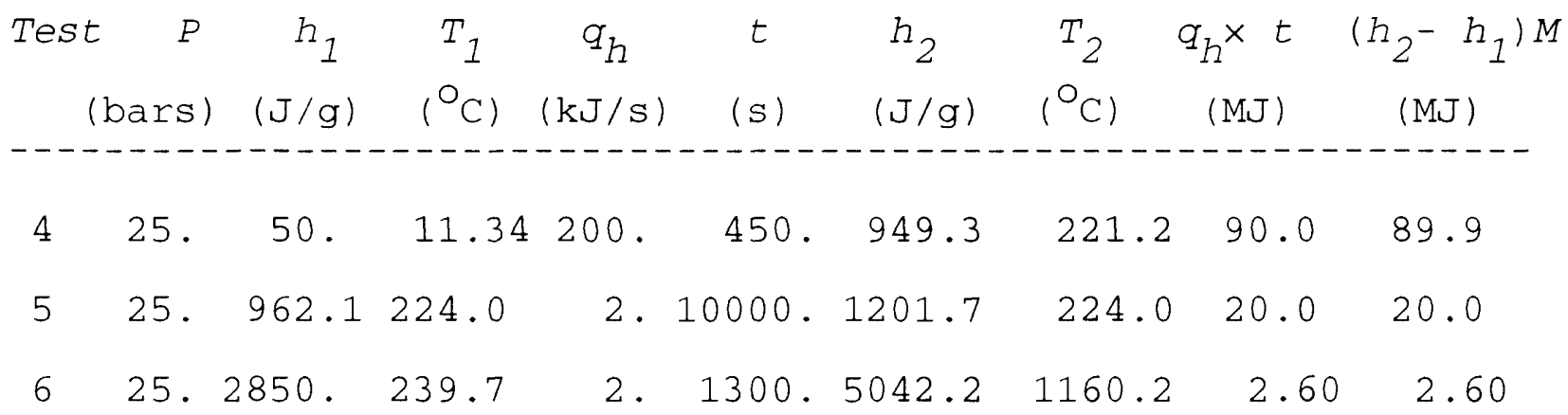


For the single-phase constant-enthalpy tests (table 7, tests 1 and 3$)$ the quantity $\left(P_{2}-P_{1}\right)$ can easily be estimated independent ly as

$$
P_{2}-P_{1}=q_{m} \times t /\left(V \rho_{f}\left[\alpha+\beta_{f}\right]\right),
$$

where $\alpha$ and $\beta_{f}$ are the compressibility of the rock and fluid, respectively. We specified a porosity $(n)$ of 1.0 , and the approximate value of $\beta_{f}$ can be derived from steam-table data (for example, Haar and others, 1984). For the conditions of the first test, the average fluid density $\left(\rho_{f}\right)$ is $947 \mathrm{~kg} / \mathrm{m}^{3}$ and $\beta_{f} \sim 6.5 \times$ $10^{-10} / \mathrm{Pa}$, so

$$
\left(P_{1}-P_{2}\right) \sim 60 /\left(947 \times\left[6.5 \times 10^{-10}\right]\right)=9.8 \times 10^{7} \mathrm{~Pa}=980 \text { bars },
$$

which is approximately the same as the numerical result. For the conditions of the third test, $\rho_{f} \sim 102 \mathrm{~kg} / \mathrm{m}^{3}$ and $\beta_{f} \sim 2.0 \times$ $10^{-8} / \mathrm{Pa}$, so

$$
\left(P_{1}-P_{2}\right) \sim 204 /\left(102 \times\left[2.0 \times 10^{-8}\right]\right)=1.0 \times 10^{8} \mathrm{~Pa}=1,000 \text { bars },
$$

again approximately the same as the numerical result.

For constant-pressure injection (table 7, tests 4-6), a 0.1 $\mathrm{m}^{3}$ volume of fluid is allowed to expand freely at constant pressure due to an energy source $q_{h}$; an arbitrarily high rock compressibility is specified. The net increase in energy $\Delta H$ is $q_{h} \times t$, and is compared with

$$
\Delta H=\left(h_{2}-h_{1}\right) M,
$$

where $M$ is the mass of fluid.

For the single-phase constant-enthalpy tests (table 7, tests 4 and 6$), \Delta H$ can also be estimated as 


$$
C_{f, P}\left(T_{1}-T_{2}\right) M
$$

where $C_{f, P}$ is the specific heat capacity at constant pressure. We specified rock heat capacity as 0.0 , and approximate values of $c_{f, P}$ can be taken from the steam tables. For the conditions of the fourth test, $M=1 \times 10^{5} \mathrm{~g}$ and $C_{f, P} \sim 4.35 \mathrm{~J} / \mathrm{g}$, so

$$
\Delta H \sim 4.35(221.2-11.3)\left(1 \times 10^{5}\right)=91.3 \mathrm{MJ},
$$

and for the conditions of the sixth test, $M=1.186 \times 10^{3} \mathrm{~g}$ and $C_{f, P} \sim 2.4 \mathrm{~J} / \mathrm{g}$, so

$$
\Delta H \sim 2.4(1160.2-239.7)\left(1.186 \times 10^{3}\right)=2.62 \mathrm{MJ} .
$$

Both values are similar to the numerical results.

\section{Steady, uniform upflow}

Bredehoeft and Papadopolous (1965) presented an analytical solution for the temperature distribution resulting from singlephase, one-dimensional, steady flow of water between constanttemperature boundaries. Posed in terms of vertical flow, their solution is

$$
\begin{gathered}
\left(T_{z}-T_{U}\right) /\left(T_{L}-T_{U}\right)=f(B, z / L) \\
\text { where } f(B, z / L)=[\exp (B z / L)-1] /[\exp (B)-1], \\
B=C_{f, P} \rho_{W} V_{D} L / K_{m^{\prime}}
\end{gathered}
$$

and $T_{U^{\prime}} T_{L^{\prime}}$ and $T_{z}$ are temperatures at the upper and lower boundaries and intermediate depths, respectively, $L$ is the distance between the constant-temperature boundaries, $C_{f, P}$ is the heat capacity of the fluid evaluated at constant pressure, $\rho_{W}$ is the density of liquid water, and $v_{D}$ is Darcian velocity. The 
dimensionless parameter $B$ is negative for upflow, positive for downflow.

For model verification it is convenient to specify a constant flowrate and use

$$
T_{z}=[f(B, z / L)]\left(T_{L}-T_{U}\right)+T_{U}
$$

to check the numerical solution for temperature. The input file included as Attachment $D$ demonstrates one way to set up HYDROTHERM for simulating upflow with constant-temperature boundaries. Figure 3 shows analytical and numerical solutions to this problem under the conditions listed in table 8. At low flow rates, there is good agreement between the analytical and numerical solutions. The assumption of constant fluid properties for the analytical solution is responsible for most of the observed disagreement. At high flowrates, the analytical solution breaks down because the upflowing fluid intercepts the boiling point-with-depth curve. Figure 3 shows, however, that the predicted temperatures for the boiling portion of the flow path agree with steam-table data.

For the results in figure 3, we used a combination of basal heat flux and fluid injection rate to hold the bottom boundary at a constant temperature across the various flow rates. The basal heat flux values, which we determined by trail and error, offset the heat lost by conduction. As table 8 illustrates, lower injection rates required greater basal heat fluxes in order to maintain a $200^{\circ} \mathrm{C}$ temperature at the bottom boundary. Attachment E demonstrates an alternative method of enforcing a constanttemperature (but not constant-pressure) lower boundary. Here, we added a constant pressure-enthalpy block at the lower boundary. Assigning this block a very low permeability and a very high thermal conductivity effectively controls the the temperature, but not the pressure, of the overlying block. The 


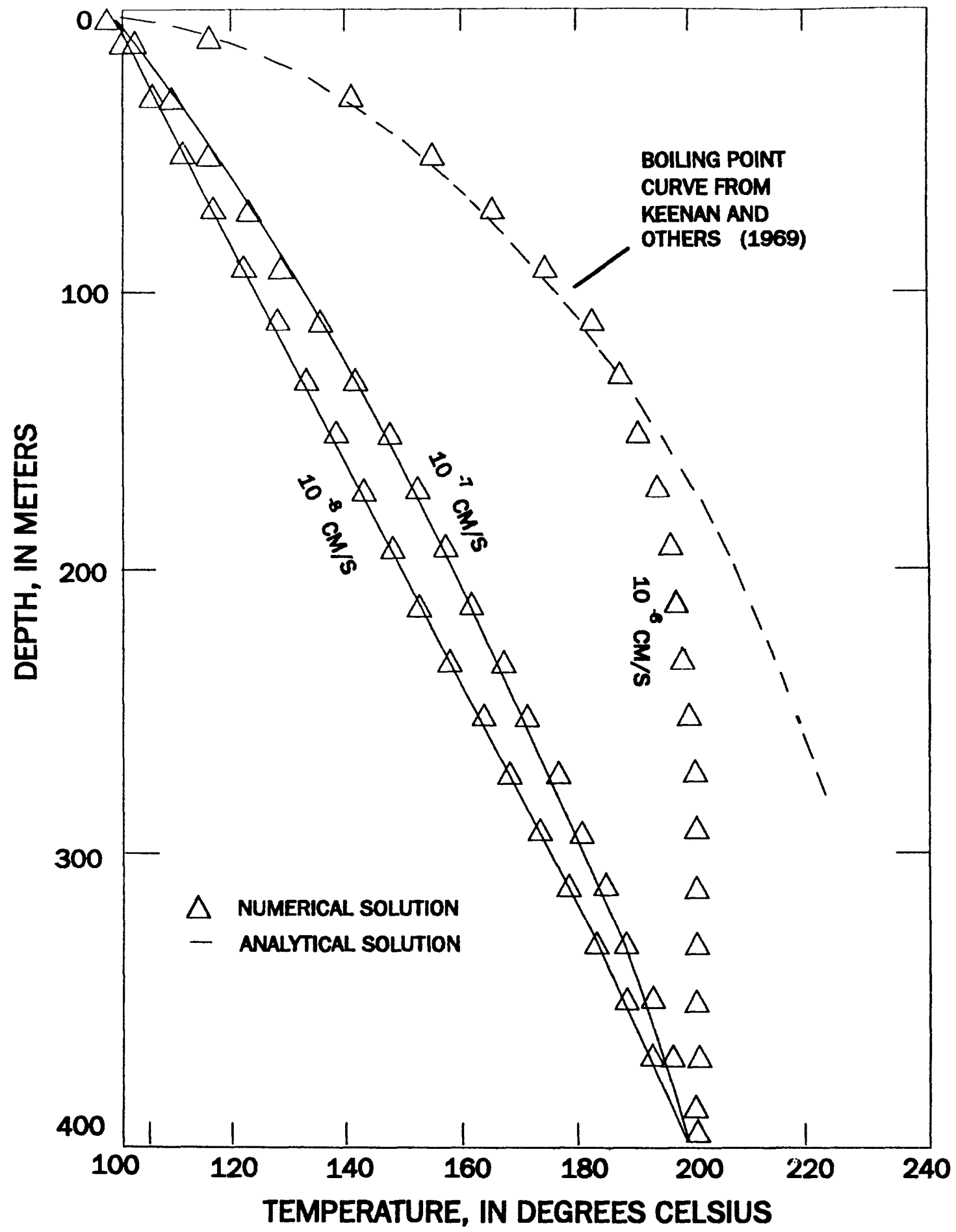

Figure 3. Temperature solutions for steady upflow at various flowrates, showing relation between numerical and analytical results. 
Table 8. Parameter values for solutions to the steady upflow problem

Values denoted by "a" are required for the analytical solution only; values denoted by " $n$ " are required for the numerical solution only. Other parameters for the numerical solutions are Iisted in Attachments $D$ and $E$

\section{For all flowrates:}

$\begin{array}{ll}\text { upper temperature }\left(T_{u}\right) & 100^{\circ} \mathrm{C} \\ \text { lower temperature }\left(T_{I}\right) & 200^{\circ} \mathrm{C} \\ \text { thermal conductivity }\left(K_{m}\right) & 2.0 \times 10^{5} \mathrm{erg} / \mathrm{sec} / \mathrm{cm} / \mathrm{K} \\ \text { distance between boundaries }(L) & 400 \mathrm{~m} \\ \text { fluid heat capacity }\left(C_{f, P}\right) & 4.186 \mathrm{~J} / \mathrm{g} / \mathrm{K} \\ \text { porosity }(n) & 0.1 \\ \text { permeability }\left(k_{Z}\right) & 1.0 \times 10^{-9} \mathrm{~cm}^{2} \\ \text { enthalpy of } 200^{\circ} \mathrm{C} \text { fluid }(h) & 8.53 \times 10^{9} \mathrm{ergs} / \mathrm{gm}\end{array}$

\section{For $10^{-8} \mathrm{~cm} / \mathrm{s}$ flowrate:}

average density of fluid $(\rho)$

$$
\begin{aligned}
& 0.92 \mathrm{~g} / \mathrm{cm}^{3} \\
& 0.93 \times 10^{-8} \mathrm{~g} / \mathrm{s} \\
& 480 \mathrm{~mW} / \mathrm{m}^{2}
\end{aligned}
$$

\section{For $10^{-7} \mathrm{~cm} / \mathrm{s}$ flowrate:}

average density of fluid $(\rho)$

$$
\begin{aligned}
& 0.91 \mathrm{~g} / \mathrm{cm}^{3} \\
& 0.91 \times 10^{-7} \mathrm{~g} / \mathrm{s} \\
& 330 \mathrm{~mW} / \mathrm{m}^{2}
\end{aligned}
$$

basal conductive heat flux

\section{For $10^{-6} \mathrm{~cm} / \mathrm{s}$ flowrate:}

average density of fluid ( $\rho$ )

$$
\begin{aligned}
& 0.87 \mathrm{~g} / \mathrm{cm}^{3} \\
& 0.87 \times 10^{-7} \mathrm{~g} / \mathrm{s} \\
& 5 \mathrm{~mW} / \mathrm{m}^{2}
\end{aligned}
$$

basal conductive heat flux 
advantage of this method is that the temperature of the lower boundary can be set directly rather than by trial-and-error.

\section{Radial flow to a well}

Sorey (1980) specified a set of problems involving radial flow to a line sink; one involves a two-phase mixture, and the numerical solution can be compared with the semi-analytical similarity solution of O'Sullivan and Pruess (1980). The twophase problem calls for an initial pressure of 30 bars, initial temperature of $233.8^{\circ} \mathrm{C}$, initial liquid saturation of 0.65 , a porosity of 0.15 , permeability of $2.4 \times 10^{-9} \mathrm{~cm}^{2}$, and a volumetric rock heat capacity of $2,000 \mathrm{~kJ} / \mathrm{m}^{3},{ }^{\circ} \mathrm{C}$ (we assign a density of $2.0 \mathrm{~g} / \mathrm{cm}^{3}$ and a specific heat capacity of $1 \times 10^{7}$ ergs/g/K). The rock matrix is assumed to be incompressible and non-conductive. Relative permeabilities are described by Coreytype functions with residual liquid and steam saturations of 0.30 and 0.05 , respectively. The problem is solved for a constant discharge of $16.7 \mathrm{~kg} / \mathrm{s}$ from a zero-radius well within a disk with $100-\mathrm{m}$ thickness. Nodal spacing is given by

$$
r_{n}=0.5 \times 2^{(n-1) / 2}, n=1,26 \text {, }
$$

where $r$ is the radial distance to the nth block, in meters. The input file for this problem is Attachment F. In figure 4, the analytical and numerical solutions are summarized in terms of pressure and saturation histories as functions of $t / r^{2}$. The minor deviation of the saturation solution at later times may be due to weighting effects like those discussed in the next section.

\section{Expanding two-phase zone}

O'Sullivan (1980) specified a problem involving fluid production from a one-dimensional geothermal reservoir overlain by a relatively low-permeability caprock. Production reduces liquid saturation in the reservoir and causes pressures to drop 

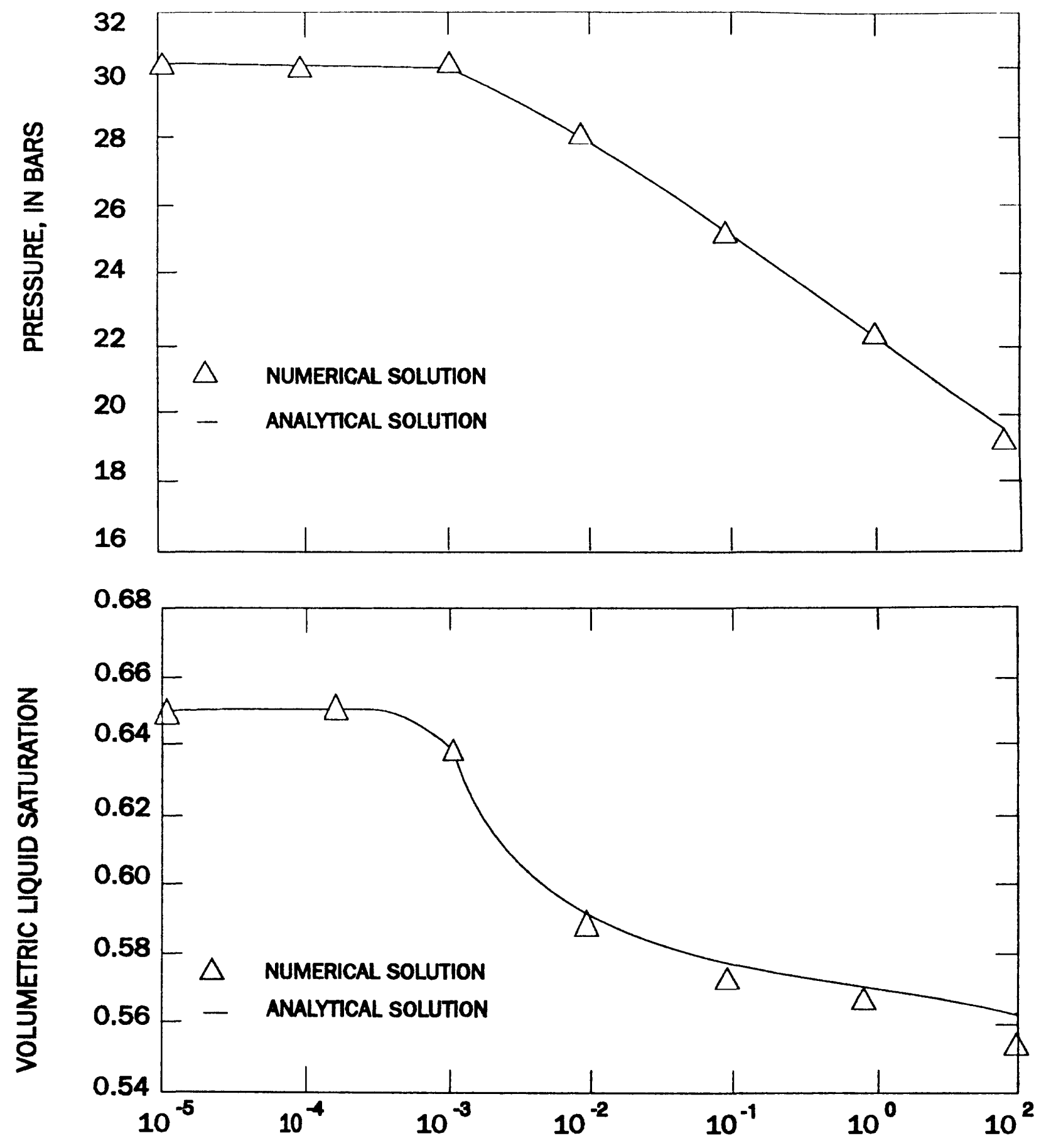

SIMULATION TIME DIVIDED BY RADIAL DISTANCE SQUARED, IN DAYS/SQUARE METER

Figure 4. Pressure and saturation solutions for steady flow to a well. HYDROTHERM results are compared with the semi-analytical solution of O'Sullivan and Pruess (1980). 
towards a vaporstatic gradient. There is no analytical solution to this problem, but we can verify the HYDROTHERM solution against published results from other numerical models (O'Sullivan, 1980).

The problem specifies a two-layer system with each layer 1 $\mathrm{km}$ thick and the bottom layer more permeable and porous $(k=1 \mathrm{x}$ $\left.10^{-9} \mathrm{~cm}^{2}, n=0.025\right)$ than the top layer $\left(k=5 \times 10^{-11} \mathrm{~cm}^{2}, n=\right.$ $0.15)$. The two layers have identical rock heat capacities (1.0 $\mathrm{kJ} / \mathrm{kg} / \mathrm{K})$ and thermal conductivities $(1.0 \mathrm{~W} / \mathrm{m} / \mathrm{K})$ and are incompressible. Initial temperatures increase linearly from $10^{\circ} \mathrm{C}$ at the land surface to $290^{\circ} \mathrm{C}$ at the interface between the two layers, and then to $310^{\circ} \mathrm{C}$ at the bottom of the lower layer. The initial pressure profile is hydrostatic for this temperature distribution. Corey-type functions describe the relative permeabilities to liquid water and steam, with residual liquid and steam saturations of 0.30 and 0.05 , respectively. The grid consists of 20 equal-sized blocks, and there is production from the lowermost block for 40 years at a rate of $100 \mathrm{~kg} / \mathrm{s} / \mathrm{km}^{2}$. The HYDROTHERM input file for this problem is Attachment $G$ to this report.

Figure 5 compares the HYDROTHERM solution for the expanding two-phase zone problem to published results from six other numerical models (O'Sullivan, 1980). The solutions from five of these six models were nearly identical; the solution for the "Intercomp" model deviated slightly.

O'Sullivan (1980) suggested that the deviation of the Intercomp solution might reflect minor inaccuracies in its description of the thermodynamic properties of water. This concerned us, because the results from HYDROTHERM are more similar to the Intercomp results than those from the other five models, which include the 1982 version, GEOTHER (Faust and Mercer, 1982). The present thermodynamic descriptions are very 


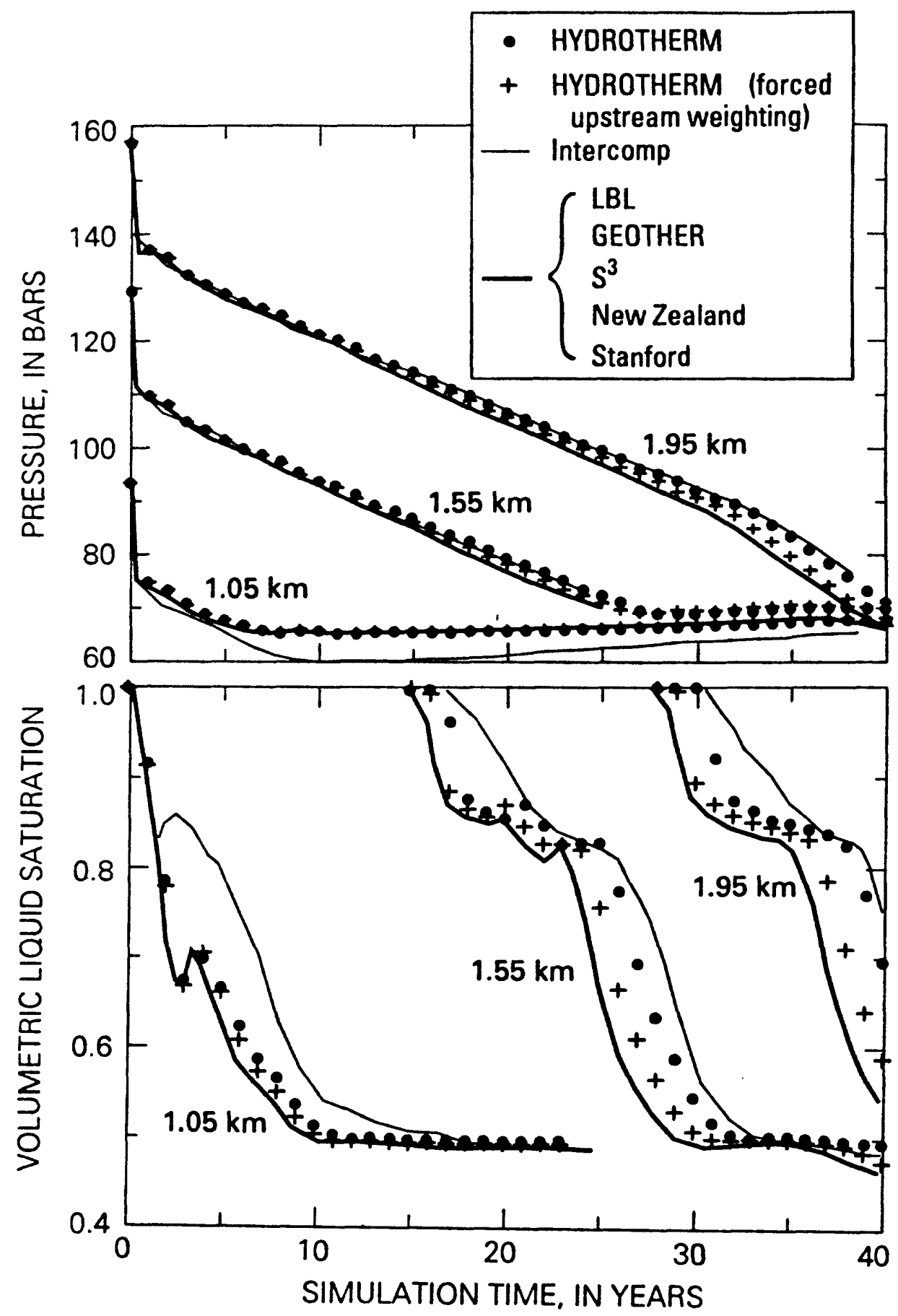

Figure 5. Pressure and saturation solutions for the expanding two-phase zone problem. HYDROTHERM results are compared with the range of numerical solutions reported by O'Sullivan (1980) for five models, including the 1982 version, GEOTHER. 
accurate, so we looked elsewhere to explain the discrepancy. One of the main differences in the numerical approach between

HYDROTHERM and GEOTHER is the method in which fluid densities and viscosities are assigned for flow between blocks. As discussed above under "Numerical approach", GEOTHER applied upstream weighting to densities and viscosities, as well as fluid enthalpies and relative permeabilities. When we forced HYDROTHERM to apply upstream weighting to viscosity and density, instead of using average values, the solution matched results from the other five models quite closely (fig. 5). Perhaps all five of these codes used upstream weighting for fluid densities and viscosities; this approach facilitates coding by allowing several parameters to be lumped and treated in the same way.

\section{Three-dimensional flow}

This problem was specified by Pruess (1980), and involves fluid production from a $4 \times 5 \times 1.8-\mathrm{km}(x, y, z)$ five-layer system. The rate of fluid production is increased stepwise three times. System response involves an expanding two-phase zone, quasisteady pressures between production increases and, at later times, recharge from three of the boundaries. The initial pressure distribution is hydrostatic; initial temperatures are $280^{\circ} \mathrm{C}$ in the lower four layers (layers $1-4$ ) and $160^{\circ} \mathrm{C}$ in the uppermost layer (layer 5); initial liquid saturation is 0.90 in layer 4 and 1.0 in the other layers. The upper and lower surfaces and the lateral boundary at $x=5 \mathrm{~km}$ are constant pressure/enthalpy boundaries. The other boundaries are no-flow boundaries. Corey-type functions describe the relative permeabilities, with residual liquid and steam saturations of 0.30 and 0.10 , respectively. Block lengths are $1.0 \mathrm{~km}$ in the $x-$ direction and $0.8 \mathrm{~km}$ in the $y$-direction. Layer 1 is $0.6-\mathrm{km}$ thick and layers 2-5 are $0.3-\mathrm{km}$ thick. Table 9 lists other problem specifications, and the input file for HYDROTHERM is Attachment $\mathrm{H}$ to this report. 
Table 9. Specifications for the three-dimensional flow problem

\section{Rock properties}

\section{Layer 1 Layer 2 Layer 3 Layer 4 Layer 5}

$\begin{array}{lllllll}x \text {-permeability } & \left(\mathrm{m}_{2}^{2}\right) & 1 \times 10^{-13} & 2 \times 10^{-13} & 2 \times 10^{-13} & 2 \times 10^{-13} & 1 \times 10^{-13} \\ y \text {-permeability } & \left(\mathrm{m}_{2}^{2}\right) & 1 \times 10^{-13} & 2 \times 10^{-13} & 2 \times 10^{-13} & 2 \times 10^{-13} & 1 \times 10^{-13} \\ z \text {-permeability } & \left(\mathrm{m}^{2}\right) & 2 \times 10^{-15} & 5 \times 10^{-14} & 5 \times 10^{-14} & 5 \times 10^{-14} & 2 \times 10^{-15} \\ \text { porosity } & & 0.20 & 0.25 & 0.25 & 0.25 & 0.25\end{array}$

The layers have uniform rock densities $\left(2,500 \mathrm{~kg} / \mathrm{m}^{3}\right)$, heat capacities (1.0 kJ/kg), and thermal conductivities $(1.0 \mathrm{~W} / \mathrm{m} / \mathrm{K}$ ) and are incompressible

\section{Initial conditions}

Layer 4 $P_{4}^{O}=P_{\text {Sat }}\left(280^{\circ} \mathrm{C}\right)$ with $S^{\circ}=0.10$

Layer $5 \quad P_{5}^{O}=P_{4}^{O}-\left(1,470 \mathrm{~m}^{2} / \mathrm{s}^{\mathrm{S}}\right) \times\left(\rho_{W, 4}^{O}+\rho_{W, 5}^{O}\right) ; T_{5}^{O}=160^{\circ} \mathrm{C}$

Layer $3 P_{3}^{O}=P_{4}^{O}+\left(1,470 \mathrm{~m}^{2} / \mathrm{s}^{\mathrm{S}}\right) \times\left(\rho_{W, 4}^{\circ}+\rho_{W, 3}^{O}\right) ; T_{3}^{O}=280^{\circ} \mathrm{C}$

Layer $2 P_{2}^{O}=P_{3}^{O}+\left(1,470 \mathrm{~m}^{2} / \mathrm{s}^{\mathrm{S}}\right) \times\left(\rho_{W, 3}^{O}+\rho_{W, 2}^{O}\right) ; T_{2}^{O}=280^{\circ} \mathrm{C}$

Layer $1 \quad P_{1}^{O}=P_{2}^{O}+\left(1,470 \mathrm{~m}^{2} / \mathrm{s}^{\mathrm{S}}\right) \times\left(\rho_{W, 2}^{O}+2 \rho_{W, 1}^{O}\right) ; T_{1}^{O}=280^{\circ} \mathrm{C}$

\section{Boundary conditions}

Top boundary

Bottom boundary
$P_{\text {top }}=P_{5}^{O}-\left(1,470 \mathrm{~m}^{2} / \mathrm{s}^{2}\right) \times \rho_{W, 5^{\circ}}^{\circ} \mathrm{T}=100^{\circ} \mathrm{C}$

$P_{\text {bot }}=P_{1}^{O}+\left(2,940 \mathrm{~m}^{2} / \mathrm{s}^{2}\right) \times \rho_{W, 1}^{O} ; T=280^{\circ} \mathrm{C}$

Maintain initial distributions of $P, T$, and $S$ along plane at $x=$ $5 \mathrm{~km}$; treat remaining lateral boundaries as closed and insulated

\section{Production schedule}

$1,000 \mathrm{~kg} / \mathrm{s}$ for $t \leq 2$ years

$2,500 \mathrm{~kg} / \mathrm{s}$ for 2 years $\leq t \leq 4$ years

$4,000 \mathrm{~kg} / \mathrm{s}$ for 4 years $\leq t \leq 6$ years

$6,000 \mathrm{~kg} / \mathrm{s}$ for $t>6$ years

All production is taken from a single corner block at $x=0.5 \mathrm{~km}$ in layer 2 
Figure 6 shows the HYDROTHERM solution to the threedimensional-flow problem compared with published results from other numerical models (Pruess, 1980). We could not run this simulation past about 6.4 years, at which time the pressure in the block representing the well dropped below 0.5 bars, the lower limit of the lookup table for the properties of water. Prior to 6.4 years the results closely match those from the other models, except for the Intercomp model. Pruess (1980) suggested that the divergence of the Intercomp solution may be due to an incorrectly specified lower boundary condition, as evidenced by high pressures below the well block. Unlike the "Expanding two-phase zone" problem, there are no apparent differences between the results from HYDROTHERM and those from the 1982 version, GEOTHER. 

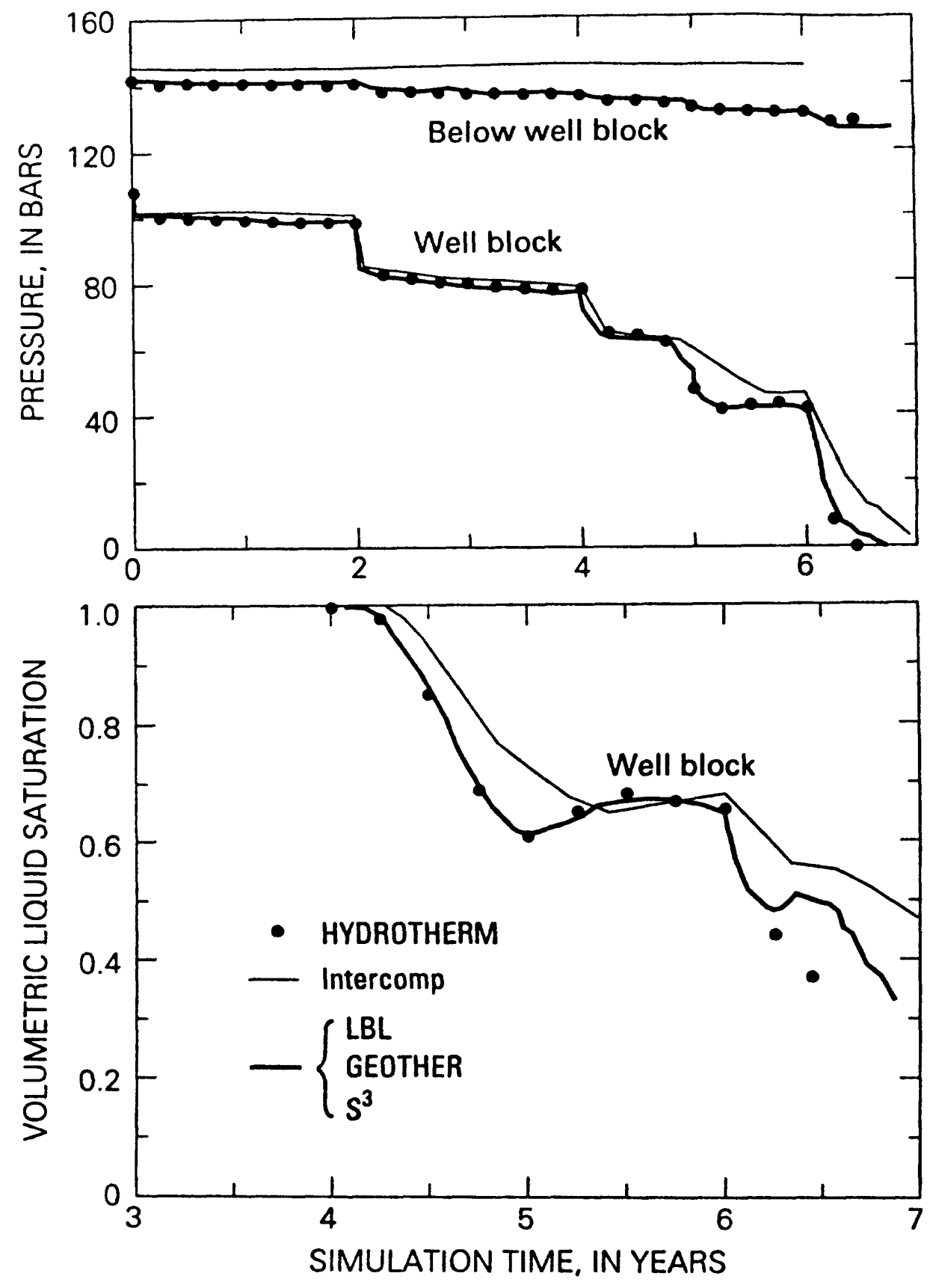

Figure 6. Pressure and saturation solutions for the threedimensional flow problem. HYDROTHERM results are compared with the range of numerical solutions reported by Pruess (1980) for four models, including the 1982 version, GEOTHER. 


\section{REFERENCES CITED}

Bear, Jacob, 1972, Dynamics of fluids in porous media: New York, American Elsevier Publishing Co., Inc., 764 p.

Bredehoeft, J.D., and Papadopolous, I.S., 1965, Rates of vertical groundwater movement estimated from the earth's thermal profile: Water Resources Research, v. 1, p. 325-328.

Cathles, L.M., 1977, An analysis of the cooling of intrusives by ground-water convection which includes boiling: Economic Geology, v. 72, p. 804-826.

Corey, A.T., 1957, Measurement of water and air permeability in unsaturated soil: Soil Science Society Proceedings, v. 21 , p. $7-10$.

Faust, C.R., and Mercer, J.W., 1977, Finite-difference model of two-dimensional, single- and two-phase heat transport in a porous medium-version I: U.S. Geological Survey Open-File Report 77-234, $84 \mathrm{p}$.

Faust, C.R., and Mercer, J.W., 1979a, Geothermal reservoir simulation 1. Mathematical models for liquid- and vapordominated hydrothermal systems: Water Resources Research, v. 15, p. 23-30.

Faust, C.R., and Mercer, J.W., 1979b, Geothermal reservoir simulation 2. Numerical solution techniques for liquid- and vapor-dominated hydrothermal systems: Water Resources Research, v. 15, p. 31-46.

Faust, C.R., and Mercer, J.W., 1982, Finite-difference model of three-dimensional, single- and two-phase heat transport in a porous medium, scepter documentation and user's manual: Reston, Virginia, Geotrans, Inc., $73 \mathrm{p}$.

Furlong, K.P., Hanson, R.B., and Bowers, J.R., 1991, Modelling thermal regimes in Kerrick, D.M., ed., Contact metamorphism: Reviews in Mineralogy, v. 26, p. 437-505.

Gladwell, I., and Wait, R., 1979, A survey of numerical methods for partial differential equations: Oxford, Clarendon Press, $434 \mathrm{p}$. 
Grant, M.A., and Sorey, M.L., 1979, The compressibility and hydraulic diffusivity of a water-steam flow: water Resources Research, v. 15, p. 684-686.

Haar, Lester, Gallagher, J.S., and Kell, G.S., 1984, NBS/NRC steam tables: thermodynamic and transport properties and computer programs for vapor and liquid states of water in SI units: New York, Hemisphere Publishing Corp., 320 p. Hanson, R.B., 1992, Effects of fluid production on fluid flow during regional and contact metamorphism: Journal of Metamorphic Geology, v. 10, p. 87-97.

Hayba, D.O., 1993, Hydrologic modeling of the Creede epithermal ore-forming system, Colorado: Ph.D. thesis, University of Illinois, $186 \mathrm{p}$.

Ingebritsen, S.E., 1986, Vapor-dominated zones within hydrothermal convection systems: Evolution and natural state: Ph.D thesis, Stanford University, $179 \mathrm{p}$. Intera Environmental Consultants, Inc., 1983, Geother: A twophase fluid flow and heat transport code: Office of Nuclear Waste Isolation Technical Report ONWI-434, $154 \mathrm{p}$. Johnson, J.W., and Norton, Denis, 1991, Critical phenomena in hydrothermal systems: state, thermodynamic, electrostatic, and transport properties of $\mathrm{H}_{2} \mathrm{O}$ in the critical region: American Journal of Science, v. 291, p. 541-648. Keenan, J.H., Keyes, F.G., Hill, P.G., and Moore, J.G., 1969, Steam tables: New York, John Wiley and Sons, $161 \mathrm{p}$. Norton, Denis, and Knight, J., 1977, Transport phenomena in hydrothermal systems: cooling plutons: American Journal of Science, v. 277, p. 937-981.

O'Sullivan, M.J., 1980, The D.O.E. code comparison project: Summary of results for problem 4 - expanding two-phase system with drainage: Proceedings Special Panel on Geothermal Model Intercomparison Study, Sixth Stanford Geothermal Workshop, p. 49-54. 
O'Sullivan, M.J., and Pruess, Karsten, 1980, Analysis of injection testing of geothermal reservoirs: Geothermal Resources Council Transactions, V. 4, p. 401-404.

Press, W.H., Flannery, B.P., Teukolsky, S.A., and Vetterling, W.T., 1986, Numerical recipes: the art of scientific computing: New York, Cambridge University Press, 818 p. Pruess, Karsten, 1980, DOE-project on geothermal reservoir engineering computer code comparison and validation, evaluation of results for problem 6: Proceedings Special Panel on Geothermal Model Intercomparison Study, Sixth Stanford Geothermal Workshop, p. 75-81.

Pruess, Karsten, 1991a, TOUGH2-A general-purpose numerical simulator for multiphase fluid and heat flow: Lawrence Berkeley Laboratory Report LBL-29400, $102 \mathrm{p}$.

Pruess, Karsten, 1991b, Grid orientation and capillary pressure effects in the simulation of water injection into depleted vapor-dominated zones: Geothermics, v. 20, p. 257-277. Roberts, P.J., Lewis, R.W., Carradori, G., and Peano, A., 1987, An extension of the thermodynamic domain of a geothermal reservoir simulator: Transport in Porous Media, v. 2, p. $397-420$.

Sass, J.H., Lachenbruch, A.H., Moses, T.H., Jr., and Morgan, Paul, 1992, Heat flow from a scientific research well at Cajon Pass, California: Journal of Geophysical Research, v. 97, p. 5,017-5,030.

Sengers, J.V., and Kamgar-Parsi, B., 1984, Representative equations for the viscosity of water substance: Journal of Physical and Chemical Reference Data, v. 13, p. 185-205. Sorey, M.L., 1980, The D.O.E. code comparison project: Summary of results for problem 2 - radial flow to a well under single and two-phase conditions: Proceedings Special Panel on Geothermal Model Intercomparison Study, Sixth Stanford Geothermal Workshop, p. 35-42. 
Sorey, M.L., Grant, M.A., and Bradford, Elizabeth, 1980, Nonlinear effects in two-phase flow to wells in geothermal reservoirs: Water Resources Research, v. 16, p. 767-777.

Watson, J.T.R., Basu, R.S., and Sengers, J.V., 1980, An improved representative equation for the dynamic viscosity of water substance: Journal of Physical and Chemical Reference Data, v. 9, p. 1,255-1,290.

White, D.E., Muffler, L.J.P., and Truesdell, A.H., 1971, Vapordominated hydrothermal systems compared with hot-water systems: Economic Geology, v. 66, p. 75-97. 


\section{ATTACHMENT A. DESCRIPTION OF SELECTED PROGRAM VARIABLES}

Wherever possible, variable names were assigned so that letters correspond to the meanings listed below. For example, the array element ZVDGW(MZM) is the $z$-direction viscositydensity-gravity term for liquid water at the minus-z-direction interface of a given $i, j, k$ block. Variables beginning with letters I to $\mathrm{N}$ are integer variables, and all other variables are double-precision real variables.

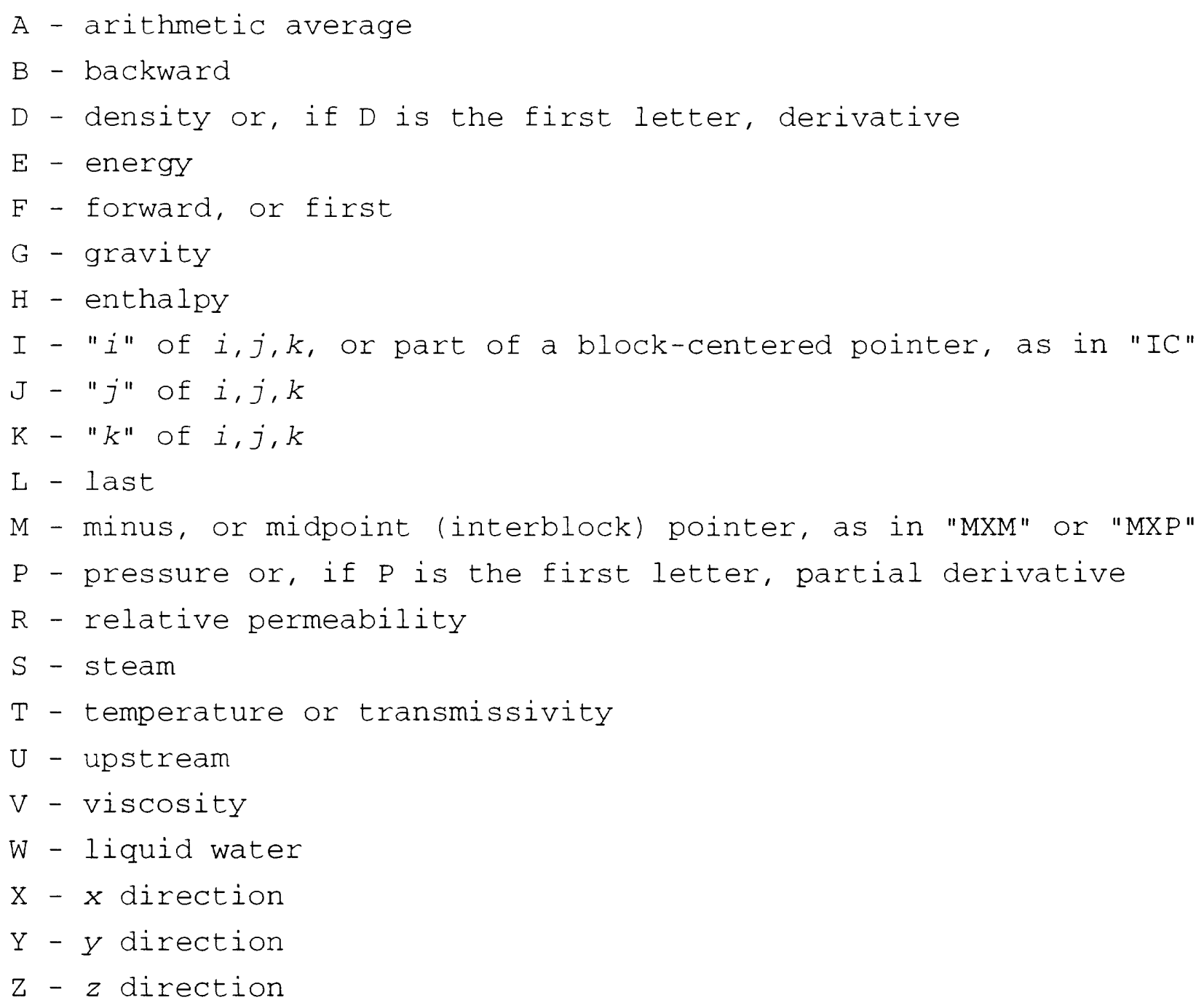

Suffixes for variables from previous time steps or NewtonRaphson iterations are: 
OLDT - added to a variable name to indicate value from the previous time step and

OLDNR - added to a variable name to indicate value from the previous Newton-Raphson iteration.

Despite the fact that HYDROTHERM is a three-dimensional model, most of the arrays are one-dimensional. This saves space and allows the program to run faster. Pointers are used to relate elements in the one-dimensional arrays to the $i, j, k$ indices of finite-difference blocks. In HYDROTHERM, the names of the various pointers have been standardized throughout. In the following equations, $\mathrm{NX}$ is the number of blocks in the $x$ direction and $\mathrm{NXX}=\mathrm{NX}+1$. Analogous relations apply in the $y$ and $z$ directions.

Pointers for block centers in 3 dimensions are:

$$
\begin{aligned}
i, j, k \text { block } & I C=(K-1) * N X+I+(J-1) * N X * N Z \\
x+1 \text { block } & I C X P=I C+1 \\
x-1 \text { block } & I C X M=I C-1 \\
z+1 \text { block } & I C Z P=I C+N X \\
z-1 \text { block } & I C Z M=I C-N X \\
y+1 \text { block } & I C Y P=I C+N X * N Z \\
\text { and } y-1 \text { block } & I C Y M=I C-N X * N Z
\end{aligned}
$$

Pointers for block centers for a vertical cross-section $(x, z)$ model are:

$$
\begin{aligned}
i, k \text { block } & I K=(K-1) * N X+I \\
X+1 \text { block } & I K X P=I K+1 \\
x-1 \text { block } & I K X M=I K-1 \\
z+1 \text { block } & I K Z P=I K+N X \\
\text { and } z-1 \text { block } & \text { IKZM }=I K-N X
\end{aligned}
$$

Pointers for block centers for a $(x, y)$ model are:

$$
i, j \text { block } \quad I J=(J-1) * N X+I \text {, etc. }
$$


Pointers for the interblock region in the $x$ direction are:

$x-1 / 2$ interblock $\mathrm{MXM}=(\mathrm{K}-1) * \mathrm{NXX}+\mathrm{I}+(\mathrm{J}-1){ }^{*} \mathrm{NXX} * \mathrm{NZ}$

and $x+1 / 2$ interblock $M X P=M X M+1$.

Pointers for the interblock region in the $y$ direction are:

$y^{-1 / 2}$ interblock $M Y M=I C$

and $y+1 / 2$ interblock $\mathrm{MYP}=\mathrm{MYM}+\mathrm{NX}{ }^{\star} \mathrm{NZ}$.

Pointers for the interblock region in the $z$ direction are:

$z-1 / 2$ interblock $\mathrm{MZM}=(\mathrm{K}-1){ }^{*} \mathrm{NX}+\mathrm{I}+(\mathrm{J}-1){ }^{*} \mathrm{NX}{ }^{*} \mathrm{NZZ}$

and $z+1 / 2$ interblock $M Z P=M Z M+N X$.

Pointers for the first and last block in a row or column are:

first $x$ block ICXF $=(\mathrm{K}-1) * \mathrm{NX}+1+(\mathrm{J}-1) * \mathrm{NX} * \mathrm{NZ}$

last $x$ block $\quad I C X L=K * N X+(J-1) * N X * N Z$

first $y$ block $\quad$ ICYF $=(K-1) * N X+I$

last $y$ block $\quad \mathrm{ICYL}=(\mathrm{K}-1) * \mathrm{NX}+\mathrm{I}+(\mathrm{NY}-1) * \mathrm{NX} * \mathrm{NZ}$

first $z$ block $\quad \mathrm{ICZF}=\mathrm{I}+(\mathrm{J}-1) * \mathrm{NX} * \mathrm{NZ}$

and last $z$ block ICZL $=(N Z-1) * N X+I+(J-1) * N X{ }^{*} N Z$.

Pointers for first and last interblock in a row or column are:

first $x$ interblock $\quad M X F=(K-1){ }^{*} N X X+1+(J-1){ }^{*} N X X{ }^{*} N Z$

last $\mathrm{x}$ interblock $\mathrm{MXL}=\mathrm{K} * \mathrm{NXX}+(\mathrm{J}-1) * \mathrm{NXX} * \mathrm{NZ}$

first $y$ interblock $\quad \mathrm{MYF}=(\mathrm{K}-1) * \mathrm{NX}+\mathrm{I}$

last $y$ interblock $\quad \mathrm{MYL}=(\mathrm{K}-1) * N X+I+N Y * N X * N Z$

first $z$ interblock $\quad \mathrm{MZF}=\mathrm{I}+(\mathrm{J}-1){ }^{*} \mathrm{NX} * \mathrm{NZZ}$

and last $z$ interblock $\quad \mathrm{MZL}=\mathrm{NZ} * \mathrm{NX}+\mathrm{I}+(\mathrm{J}-1){ }^{*} \mathrm{NX}{ }^{\star N Z Z}$. 


\section{ATTACHMENT B. INPUT FILE FOR ONE-BLOCK PROBLEM: CONSTANT ENTHALPY}

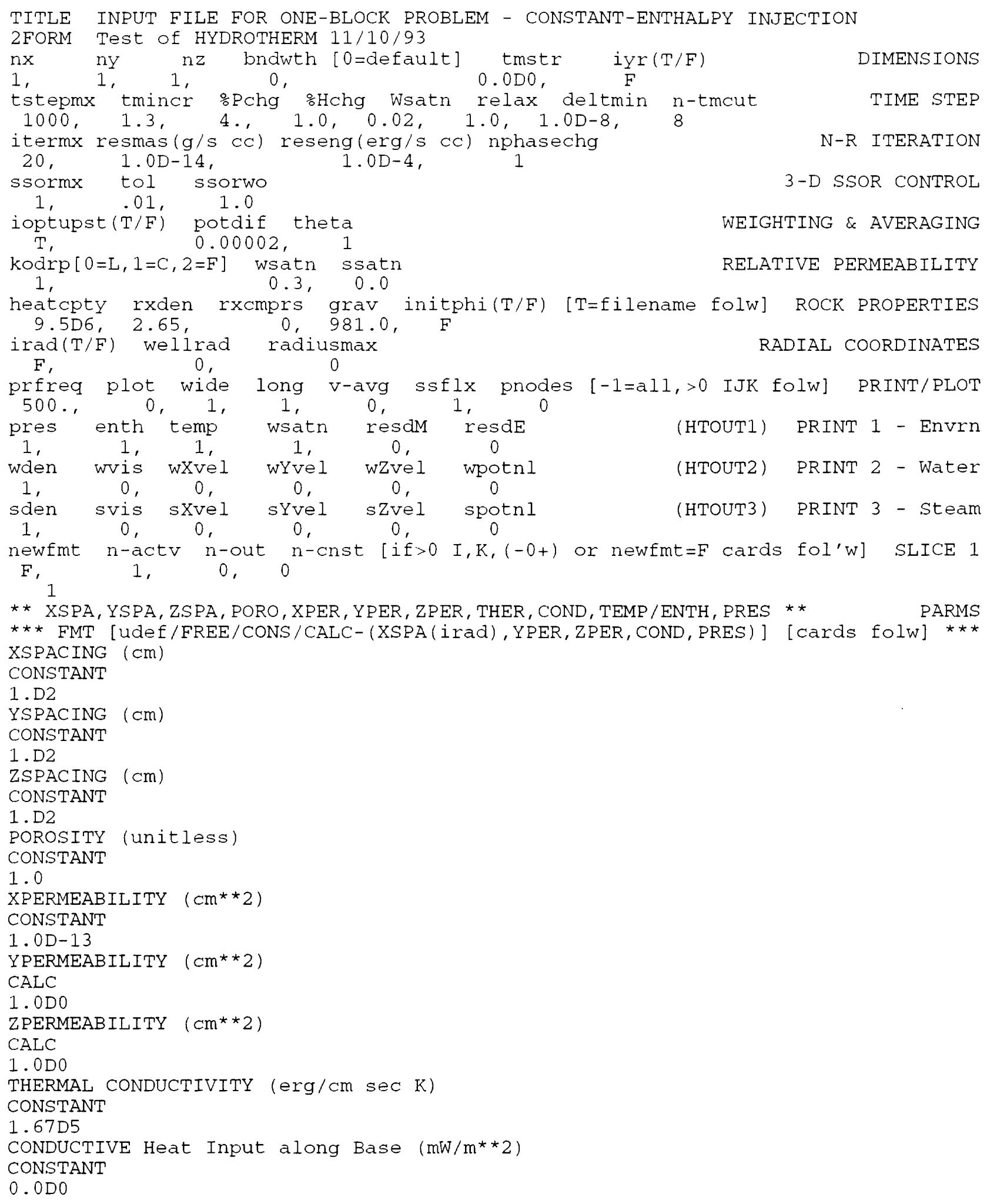


ENTHALPY

FREE

$6.32331 D 9$

PRESSURE (dynes/cm**2)

FREE

5. OD6

$\star \star *$ PERIOD, SRC/SINK (if $>0)$, PRINT OFTS (if $=\mathrm{T}), \&$ PARMS tchg $[-1=s t p]$ delt $[-1=\mathrm{NC}]$ nsrce $[-1=\mathrm{NC}]$ ichgpr nparms

(if $>0$ ) cards follow $* \star \star$

5000 , 10 ,

TIME PERIOD 1

i j 'wlflx(g/s;+=injct) wlenth(erg/g) n-openint [k's folw] SOURCE/SINK 1

$1,1, \quad 50$,

tchg $[-1=s t p]$ delt $[-1=\mathrm{NC}]$ nsrce $[-1=\mathrm{NC}]$ -1.0 ,

0 ,

0 ,

ichgpr nparms

TERMINATE RUN

F,

0 


\section{ATTACHMENT C. INPUT FILE FOR ONE-BLOCK PROBLEM: CONSTANT PRESSURE}

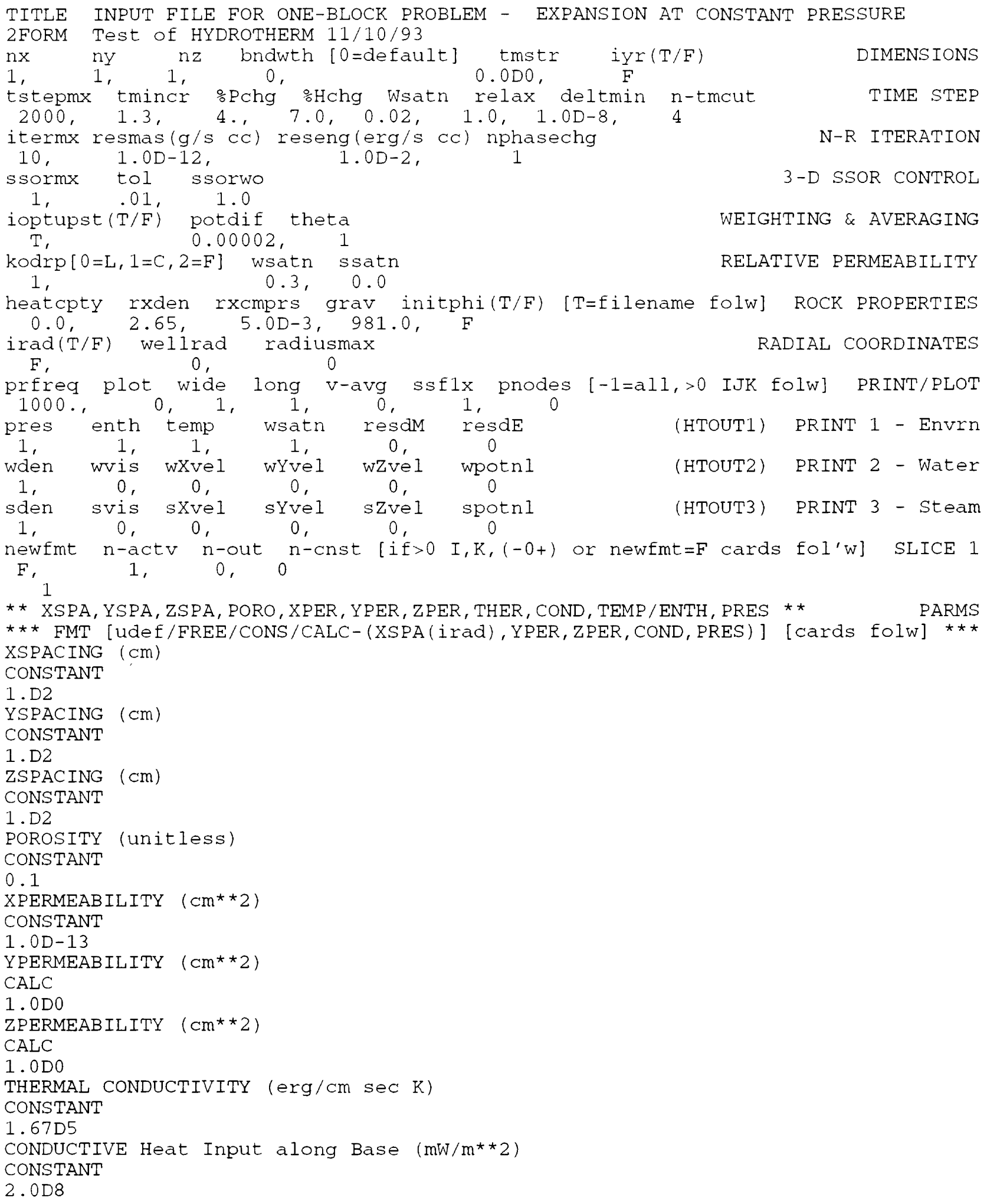




\section{ENTHALPY}

FREE

$0.5 D 9$

PRESSURE (dynes/ $\mathrm{cm} * \star 2$ )

FREE

$25.0 D 6$

$\star \star \star$ PERIOD, SRC/SINK (if $>0)$, PRINT OFTS (if $=\mathrm{T}), \&$ PARMS

tchg $[-1=s t p]$ delt $[-1=N C]$ nsrce $[-1=N C]$ ichgpr nparms

(if $>0$ ) cards follow ***

450 . ,

5 ., 0 , F, TIME PERIOD 1

tchg [-1=stp]

delt $[-1=\mathrm{NC}]$

-1.0 ,

0 ,

nsrce $[-1=\mathrm{NC}]$

ichgpr

o

F,

0

TERMINATE RUN 


\section{ATTACHMENT D. INPUT FILE FOR STEADY, UNIFORM UPFLOW: CONSTANT FLUX}

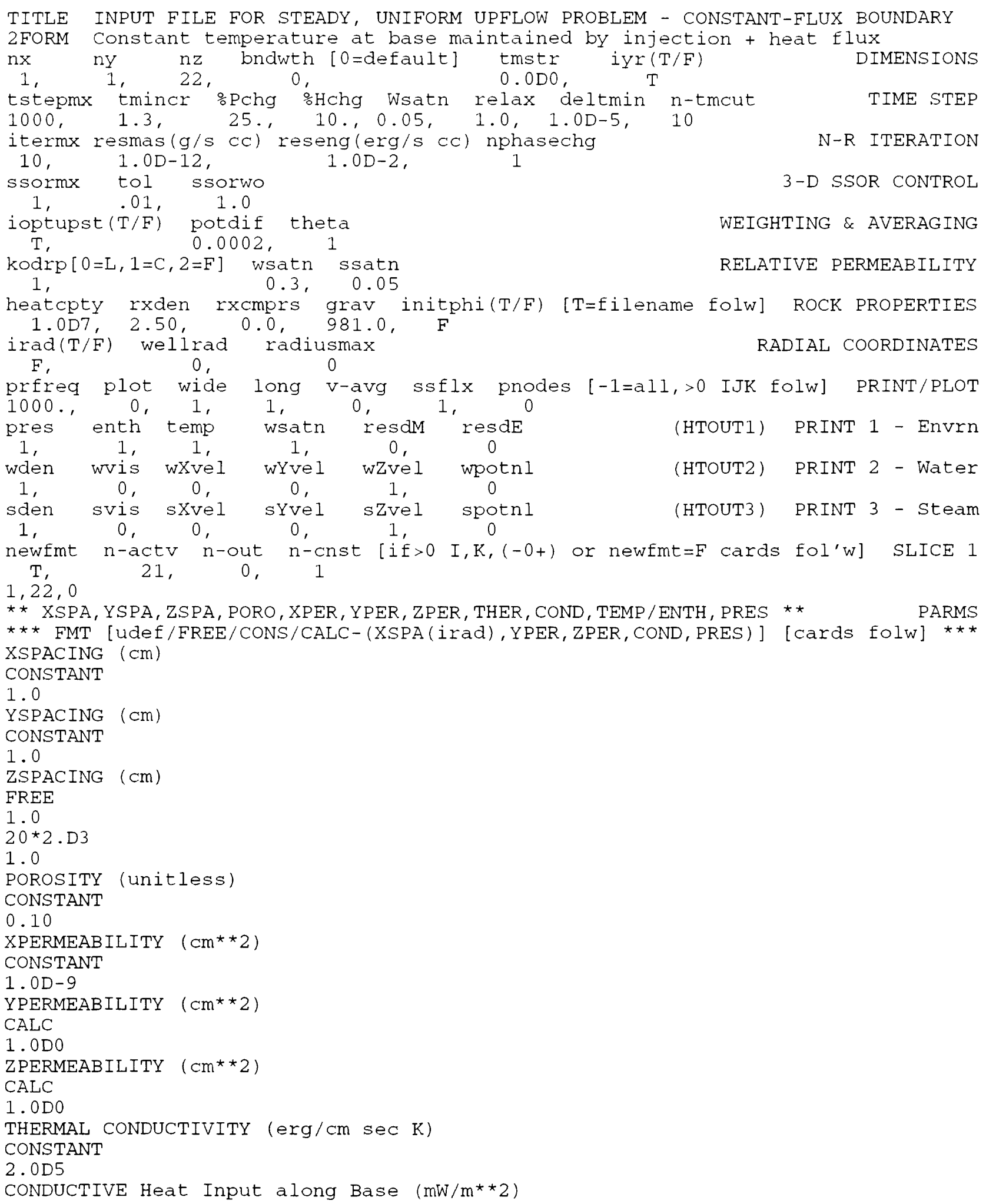


CONSTANT

480.0

PRESSURE (dynes/ $\mathrm{cm}^{\star *}$ )

CALC

1.013D6

TEMPERATURE (C)

FREE

200 .

197.5

192.5

187.5

182.5

177.5

172.5

167.5

162.5

157.5

152.5

147.5

142.5

137.5

132.5

127.5

122.5

117.5

112.5

107.5

100.0

99.99

$\star \star \star$ PERIOD, SRC/SINK (if $>0$ ), PRINT OPTS (if $=$ T), \& PARMS (if $>0$ ) cards follow *** tchg[-1=stp] delt $[-1=\mathrm{NC}]$ nsrce[-1=NC] ichgpr nparms TIME PERIOD 1

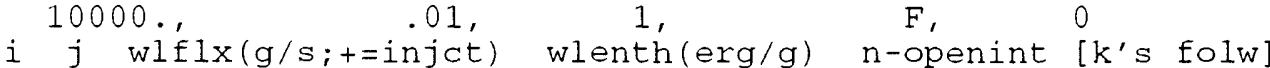
$1,1.93 \mathrm{D}-8$ 8.53D9 1 SOURCE/SINK 1 1 tchg $[-1=\mathrm{stp}]$ delt $[-1=\mathrm{NC}]$ -1.0 ,

delt $[-1=\mathrm{NC}]$ nsrce $[-1=\mathrm{NC}]$

0 ,

ichgpr nparms

TERMINATE RUN 


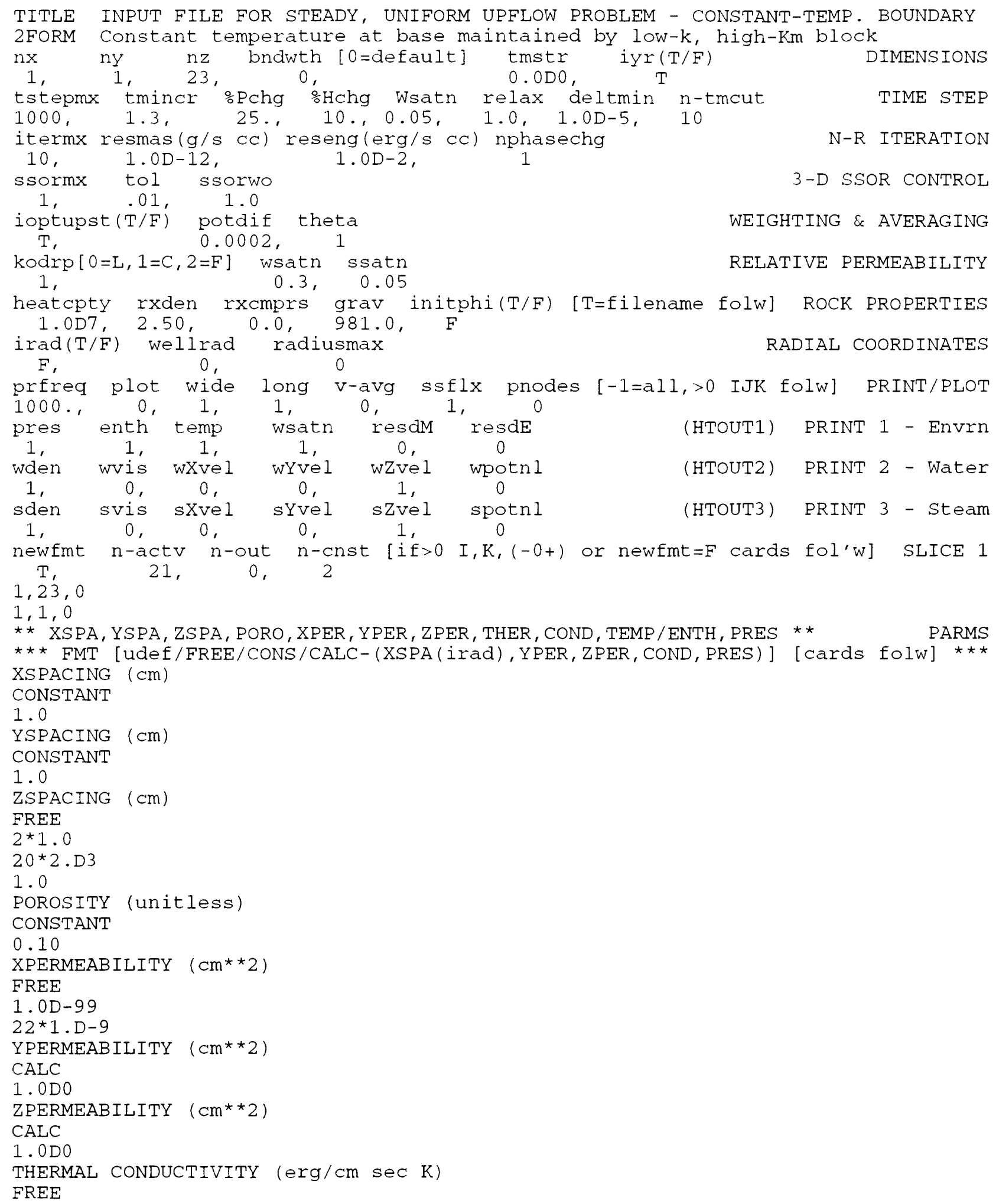


$1.0 \mathrm{D} 99$

$22 * 2.0 \mathrm{D} 5$

CONDUCTIVE Heat Input along Base $(\mathrm{mW} / \mathrm{m} * \star 2)$

CONSTANT

0.0

PRESSURE (dynes $/ \mathrm{cm} * \star 2$ )

CALC

$1.013 \mathrm{D} 6$

TEMPERATURE (C)

FREE

200.0

200.0

197.5

192.5

187.5

182.5

177.5

172.5

167.5

162.5

157.5

152.5

147.5

142.5

137.5

132.5

127.5

122.5

117.5

112.5

107.5

100.0

99.99

*** PERIOD, SRC/SINK (if $>0$ ), PRINT OPTS (

tchg $[-1=s t p]$ delt $[-1=N C]$ nsrce $[-1=N C$ 10000 . .01 ,

i j wlflx (g/s;+=injct) wlenth(erg/g) $1,1 \quad .93 \mathrm{D}-8$

8.53D9

ichgpr \& PARMS

(if $>0$ ) cards follow ***

tchg $[-1=\operatorname{stp}]$ delt $[-1=\mathrm{NC}]$ nsrce $[-1=\mathrm{NC}]$ ichgpr nparms TIME PERIOD 1 SOURCE/SINK 1

-1.0 ,

0 ,

0 ,

F,

TERMINATE RUN 


\section{ATTACHMENT F. INPUT FILE FOR RADIAL FLOW TO A WELL}

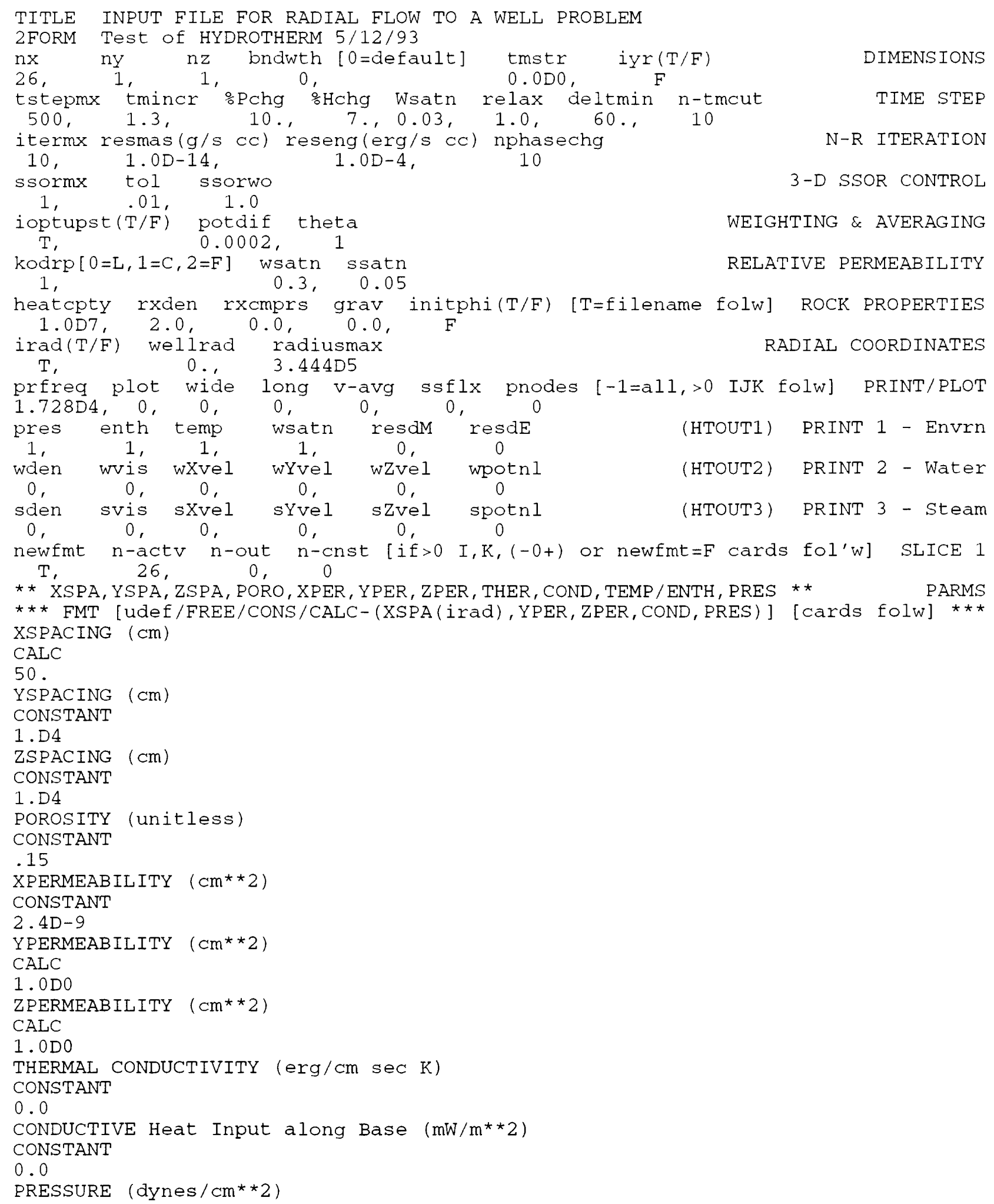


CONSTANT

3.D7

ENTHALPY (ergs/g)

CONSTANT

$1.0260 \mathrm{E} 10$

** PERIOD, SRC/SINK (if $>0)$, PRINT OPTS

tchg [-1=stp] delt [-1=NC] nsrce [-1=NC]

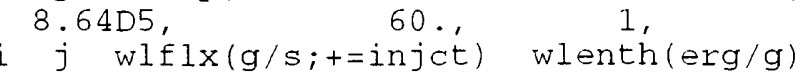
60.

if $=T), \&$ PARMS

(if $>0$ ) cards follow $* * *$ $1,1-1.67 D 4$

0.0

ichgpr nparms

TIME PERIOD 1

1

tchg $[-1=\operatorname{stp}]$

-1.0 ,

$\operatorname{delt}[-1=\mathrm{NC}]$

nsrce $[-1=\mathrm{NC}]$

$\mathrm{F}$, 0

$\mathrm{n}$-openint [k's folw] 1

SOURCE/SINK 1

ichgpr nparms

$\mathrm{F}, \quad 0$

TERMINATE RUN

0 , 


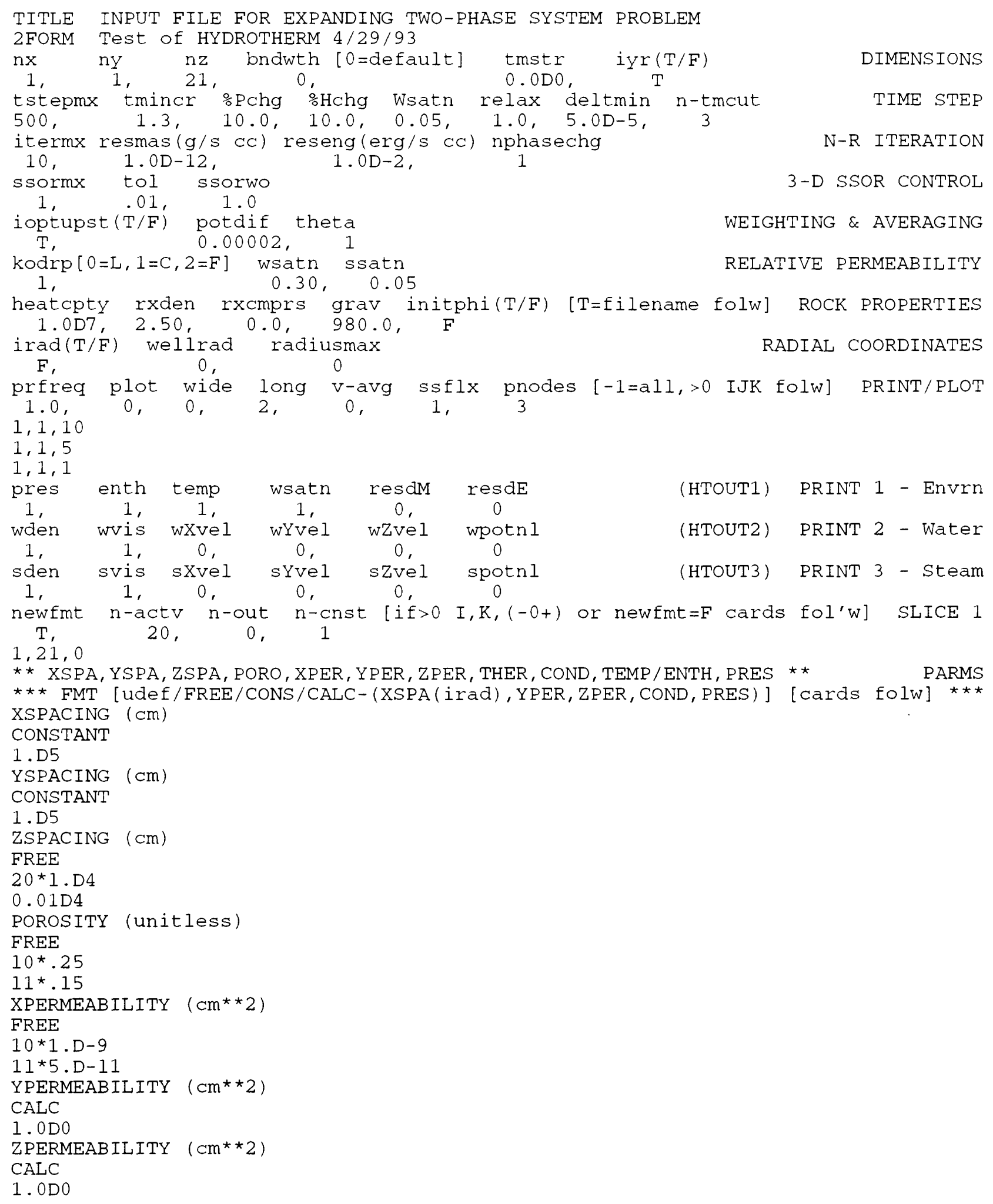




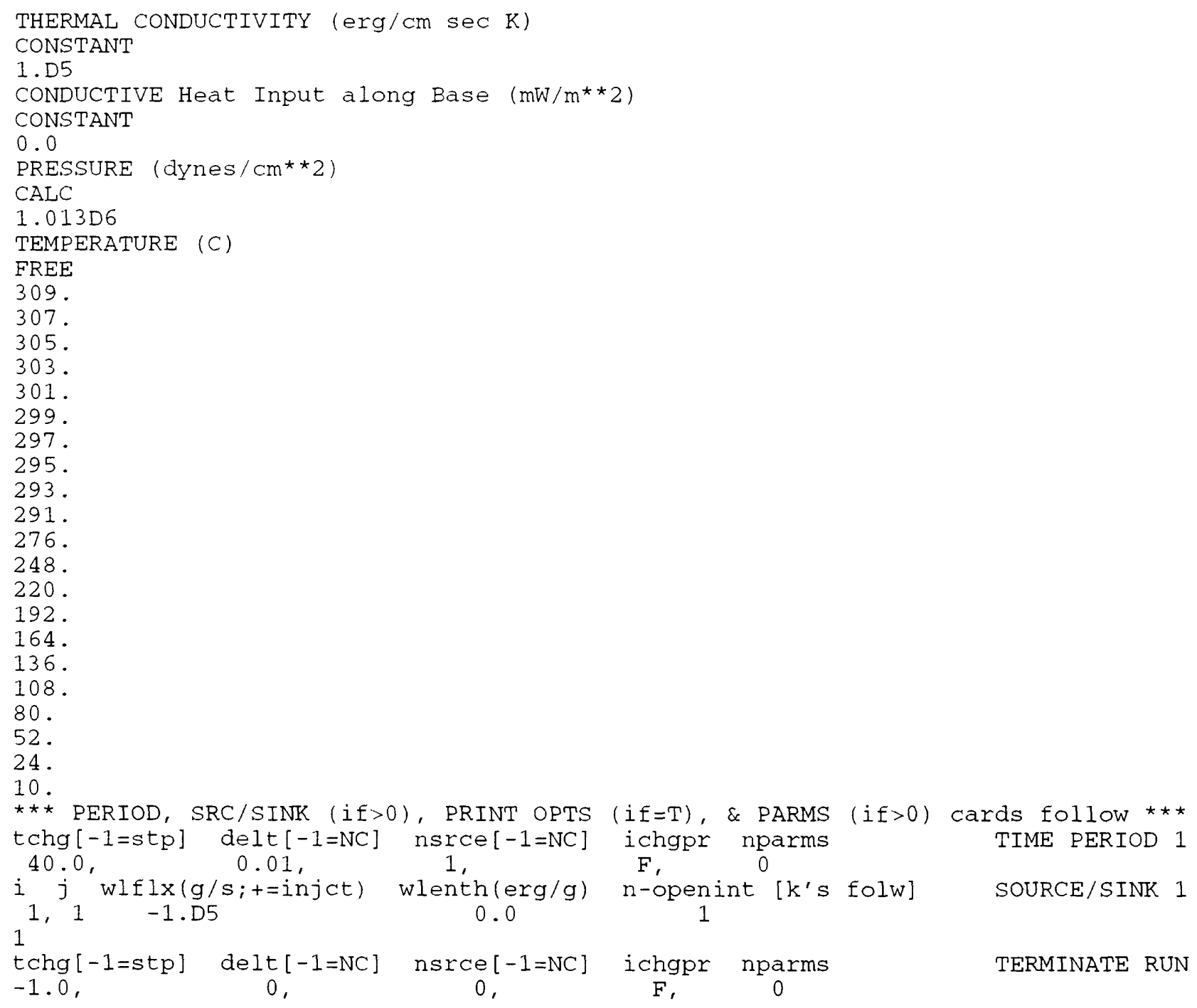




\section{ATTACHMENT H. INPUT FILE FOR THREE-DIMENSIONAL FLOW PROBLEM}

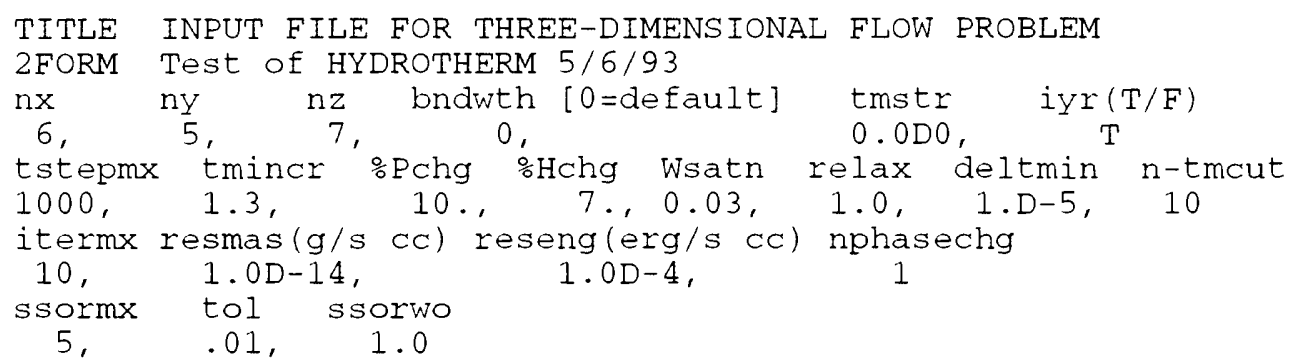

DIMENSIONS

TIME STEP

N-R ITERATION

3-D SSOR CONTROL WEIGHTING \& AVERAGING

RELATIVE PERMEABILITY

$\operatorname{kodrp}[0=L, 1=C, 2=F]$ wsatn ssatn

$$
1, \quad 0.3,0.10
$$

heatcpty rxden rxcmprs grav initphi(T/F) [T=filename folw] ROCK PROPERTIES 1.0D7, 2.50, $0.0, \quad 981.0, \quad \mathrm{~F}$

irad $(\mathrm{T} / \mathrm{F})$ wellrad radiusmax

RADIAL COORDINATES

prfireq plot wide long $v$-avg ssflx pnodes $[-1=a 11,>0$ IJK folw] PRINT/PLOT $0.25, \quad 0,0,1, \quad 0,1, \quad 0$

pres enth temp wsatn resdM resdE

$1,1,0,1, \quad 0, \quad 0$

wden wris wXvel wYvel wZvel wpotnl

(HTOUT1) PRINT 1 - Envrn

$1, \quad 0, \quad 0, \quad 0, \quad 0, \quad 0$

sden svis sXvel sYvel sZvel spotnl (HTOUT3) PRINT 3 - Steam

$1,0,0,0,0$

$K,(-0+)$ or newfmt $=F$ cards fol'w] SLICE 1

$\begin{array}{crrr}\text { newfmt } & \text { n-actv } & \text { n-out } & n \text {-cn } \\ \text { F, } & 25, & 2, & 15\end{array}$

$\begin{array}{llllll}-1 & -1 & -1 & -1 & -1 & 0\end{array}$

$\begin{array}{llllll}1 & 2 & 3 & 4 & 5 & -1\end{array}$

$\begin{array}{llllll}6 & 7 & 8 & 9 & 10 & -1\end{array}$

$\begin{array}{llllll}11 & 12 & 13 & 14 & 15 & -1\end{array}$

$\begin{array}{llllll}16 & 17 & 18 & 19 & 20 & -1\end{array}$

$\begin{array}{llllll}21 & 22 & 23 & 24 & 25 & -1\end{array}$

$\begin{array}{llllll}-1 & -1 & -1 & -1 & -1 & 0\end{array}$

newfmt n-actv n-out n-cnst [if>0 I, $K,(-0+)$ or newfmt=F cards fol'w] SLICE 2

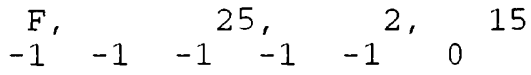

$\begin{array}{rrrrrr}-1 & -1 & -1 & -1 & -1 & 0 \\ 1 & 2 & 3 & 4 & 5 & -1\end{array}$

$\begin{array}{llllll}6 & 7 & 8 & 9 & 10 & -1\end{array}$

$\begin{array}{llllll}11 & 12 & 13 & 14 & 15 & -1\end{array}$

$\begin{array}{llllll}16 & 17 & 18 & 19 & 20 & -1\end{array}$

$\begin{array}{llllll}21 & 22 & 23 & 24 & 25 & -1\end{array}$

$\begin{array}{llllll}-1 & -1 & -1 & -1 & -1 & 0\end{array}$

newfmt n-actv n-out n-cnst [if>0 I, K, $(-0+)$ or newfmt=F cards fol'w] SLICE 3

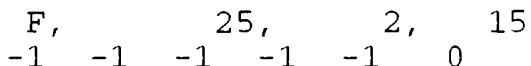

$\begin{array}{rrrrrr}-1 & -1 & -1 & -1 & -1 & 0 \\ 1 & 2 & 3 & 4 & 5 & -1\end{array}$

$\begin{array}{llllll}6 & 7 & 8 & 9 & 10 & -1\end{array}$

$\begin{array}{llllll}11 & 12 & 13 & 14 & 15 & -1\end{array}$

$\begin{array}{llllll}16 & 17 & 18 & 19 & 20 & -1\end{array}$

$\begin{array}{llllll}21 & 22 & 23 & 24 & 25 & -1\end{array}$

$\begin{array}{llllll}-1 & -1 & -1 & -1 & -1 & 0\end{array}$

newfmt n-actv n-out n-cnst [if>0 $I, K,(-0+)$ or newfmt=F cards fol'w] SLICE 4 \begin{tabular}{rrrrrr}
$F$, & \multicolumn{2}{c}{25,} & \multicolumn{2}{c}{2,} \\
-1 & -1 & -1 & -1 & -1 & 0 \\
1 & 2 & 3 & 4 & 5 & -1 \\
6 & 7 & 8 & 9 & 10 & -1
\end{tabular} 15 


$$
\begin{array}{rrrrrr}
11 & 12 & 13 & 14 & 15 & -1 \\
16 & 17 & 18 & 19 & 20 & -1 \\
21 & 22 & 23 & 24 & 25 & -1 \\
-1 & -1 & -1 & -1 & -1 & 0
\end{array}
$$

newfmt n-actv n-out $n$-cnst [if>0 I,,$(-0+)$ or newfmt=F cards fol'w] SLICE 5 F, 25, 2, 15

$\begin{array}{rrrrrr}-1 & -1 & -1 & -1 & -1 & 0 \\ 1 & 2 & 3 & 4 & 5 & -1\end{array}$

$\begin{array}{rrrrrr}1 & 2 & 3 & 4 & 5 & -1 \\ 6 & 7 & 8 & 9 & 10 & -1\end{array}$

$\begin{array}{llllll}11 & 12 & 13 & 14 & 15 & -1\end{array}$

$\begin{array}{llllll}16 & 17 & 18 & 19 & 20 & -1\end{array}$

$\begin{array}{llllll}21 & 22 & 23 & 24 & 25 & -1\end{array}$

$\begin{array}{llllll}-1 & -1 & -1 & -1 & -1 & 0\end{array}$

* * XSPA, YSPA, ZSPA, PORO, XPER, YPER, ZPER, THER, COND, TEMP/ENTH, PRES * *

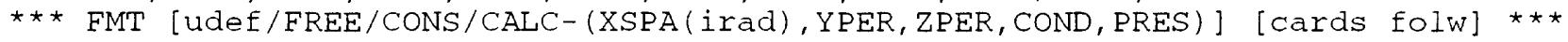
XSPACING $(\mathrm{cm})$

\section{FREE}

$5 \star 1 . D 5,1.0$

YSPACING $(\mathrm{cm})$

FREE

$5 * 8 . D 4$

ZSPACING $(\mathrm{cm})$

FREE

$1.0,6 . D 4,4 * 3 . D 4,1.0$

POROSITY (unitless)

FREE

$12 * 0.20,30 * 0.25$

$12 * 0.20,30 * 0.25$

$12 * 0.20,30 * 0.25$

$12 * 0.20,30 * 0.25$

$12 * 0.20,30 * 0.25$

XPERMEABILITY $\left(\mathrm{cm}^{\star \star} 2\right)$

FREE

$12 * 1 . D-9,18 * 2 . D-9,12 * 1 . D-9$

$12 * 1 . D-9,18 * 2 . D-9,12 * 1 . D-9$

$12 * 1 . D-9,18 * 2 . D-9,12 * 1 . D-9$

$12 * 1 . D-9,18 * 2 . D-9,12 * 1 . D-9$

$12 * 1 . D-9,18 * 2 . D-9,12 * 1 . D-9$

YPERMEABILITY $\left(\mathrm{cm}^{\star \star} 2\right)$

CALC

1.ODO

ZPERMEABILITY $(\mathrm{cm} * \star 2)$

FREE

$12 * 2 . D-11,18 * 5 \cdot D-10,12 * 2 . D-11$

$12 * 2 . D-11,18 * 5 . D-10,12 * 2 . D-11$

$12 * 2 \cdot D-11,18 * 5 \cdot D-10,12 * 2 \cdot D-11$

$12 * 2 \cdot \mathrm{D}-11,18 * 5 \cdot \mathrm{D}-10,12 * 2 \cdot \mathrm{D}-11$

$12 * 2 \cdot \mathrm{D}-11,18 * 5 \cdot \mathrm{D}-10,12 * 2 . \mathrm{D}-11$

THERMAL CONDUCTIVITY (erg/cm sec K)

CONSTANT

$1 . \mathrm{D} 5$

CONDUCTIVE Heat Input along Base $\left(\mathrm{mW} / \mathrm{m}^{* * 2}\right)$

CONSTANT

0.0

PRESSURE (dynes $/ \mathrm{cm}^{\star *} 2$ )

\section{FREE}

$6 * 164.5 \mathrm{D} 6,6 * 142.1 \mathrm{D} 6,6 * 108.5 \mathrm{D} 6,6 * 86.24 \mathrm{D} 6,6 * 64.12 \mathrm{D} 6,6 * 39.72 \mathrm{D} 6,6 * 26.35 \mathrm{D} 6$ $6 * 164.5 \mathrm{D} 6,6 * 142.1 \mathrm{D} 6,6 * 108.5 \mathrm{D} 6,6 * 86.24 \mathrm{D} 6,6 * 64.12 \mathrm{D} 6,6 * 39.72 \mathrm{D} 6,6 * 26.35 \mathrm{D} 6$ $6 * 164.5 \mathrm{D} 6,6 * 142.1 \mathrm{D} 6,6 * 108.5 \mathrm{D} 6,6 * 86.24 \mathrm{D} 6,6 * 64.12 \mathrm{D} 6,6 * 39.72 \mathrm{D} 6,6 * 26.35 \mathrm{D} 6$ $6 * 164.5 \mathrm{D} 6,6 * 142.1 \mathrm{D} 6,6 * 108.5 \mathrm{D} 6,6 * 86.24 \mathrm{D} 6,6 * 64.12 \mathrm{D} 6,6 * 39.72 \mathrm{D} 6,6 * 26.35 \mathrm{D} 6$ $6 * 164.5 D 6,6 * 142.1 D 6,6 * 108.5 D 6,6 * 86.24 D 6,6 * 64.12 D 6,6 * 39.72 D 6,6 * 26.35 D 6$ ENTHALPY $(\mathrm{erg} / \mathrm{g})$ 


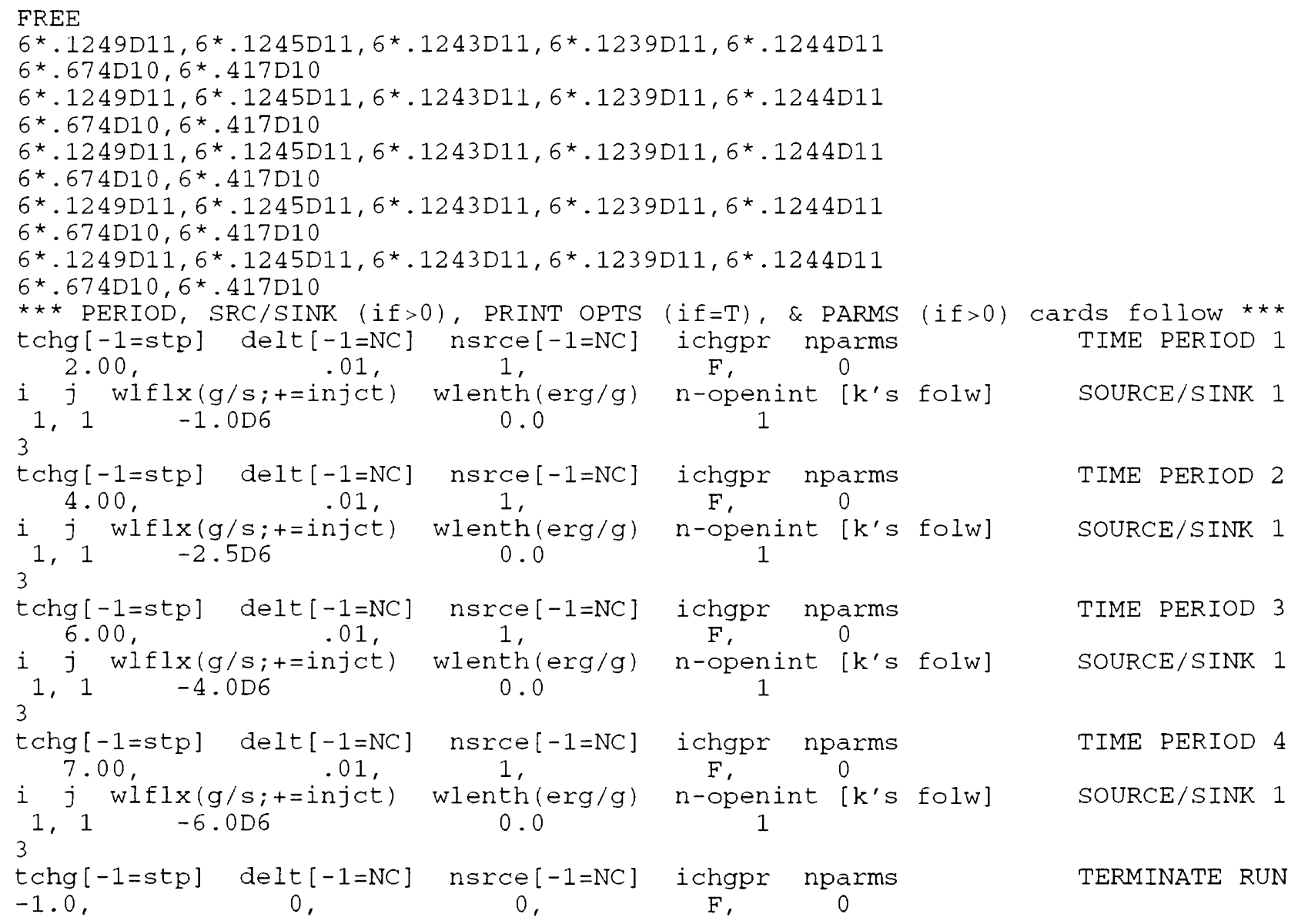

